\title{
Astrocytic tight junctions control inflammatory CNS lesion pathogenesis
}

\author{
Sam Horng, ${ }^{1,2,3}$ Anthony Therattil, ${ }^{1,2,3}$ Sarah Moyon, ${ }^{1,2,3,4}$ Alexandra Gordon, ${ }^{1,2,3}$ Karla Kim, ${ }^{1,2,3}$ Azeb Tadesse Argaw, ${ }^{1,2,3}$ Yuko Hara, ${ }^{1,4}$ \\ John N. Mariani, ${ }^{1,2,3}$ Setsu Sawai, ${ }^{1,2,3}$ Per Flodby, ${ }^{5}$ Edward D. Crandall, ${ }^{5}$ Zea Borok, ${ }^{5}$ Michael V. Sofroniew, ${ }^{6}$ Candice Chapouly, ${ }^{1,2,3}$ \\ and Gareth R. John ${ }^{1,2,3}$
}

${ }^{1}$ Friedman Brain Institute, ${ }^{2}$ Corinne Goldsmith Dickinson Center for Multiple Sclerosis, ${ }^{3}$ Department of Neurology, and ${ }^{4}$ Department of Neuroscience, Icahn School of Medicine at Mount Sinai, New York, New York, USA. ${ }^{5}$ Will Rogers Institute Pulmonary Research Center, Division of Pulmonary, Critical Care, and Sleep Medicine, Department of Medicine, Keck School of Medicine, University of Southern California, Los Angeles, California, USA. ${ }^{6}$ Neurobiology, David Geffen School of Medicine, UCLA, Los Angeles, California, USA.

\begin{abstract}
Lesions and neurologic disability in inflammatory CNS diseases such as multiple sclerosis (MS) result from the translocation of leukocytes and humoral factors from the vasculature, first across the endothelial blood-brain barrier (BBB) and then across the astrocytic glia limitans (CL). Factors secreted by reactive astrocytes open the BBB by disrupting endothelial tight junctions (TJs), but the mechanisms that control access across the GL are unknown. Here, we report that in inflammatory lesions, a second barrier composed of reactive astrocyte TJs of claudin 1 (CLDN1), CLDN4, and junctional adhesion molecule A (JAM-A) subunits is induced at the GL. In a human coculture model, CLDN4-deficient astrocytes were unable to control lymphocyte segregation. In models of CNS inflammation and MS, mice with astrocyte-specific Cldn4 deletion displayed exacerbated leukocyte and humoral infiltration, neuropathology, motor disability, and mortality. These findings identify a second inducible barrier to CNS entry at the GL. This barrier may be therapeutically targetable in inflammatory CNS disease.
\end{abstract}

\section{Introduction}

In autoinflammatory conditions such as multiple sclerosis (MS) and neuromyelitis optica (NMO), penetration into the CNS of inflammatory cells, and soluble factors such as autoantibodies, cytokines, and plasma proteins, drives lesion formation and clinical relapse $(1,2)$. New therapies that limit immune component entry are needed, as presently only high-dose corticosteroids and plasmapheresis effectively treat exacerbations in the short term, but can cause significant side effects and have minimal impact on long-term disability $(3,4)$. Identifying key mechanisms controlling leukocyte and humoral entry may lead to novel agents that more effectively limit relapse severity, as well as prevent relapses and long-term disability. Such approaches may also delay onset of the secondary progressive phase of MS, which has been linked to neurodegeneration resulting from demyelinating and neuronal injury during the relapsing-remitting stage (5).

The CNS is unique in that entry of circulating immune components is normally restricted (6). To enter the parenchyma from the vasculature, leukocytes and soluble factors must traverse 2 barriers. Initially, they must cross the blood-brain barrier (BBB), which exists at the level of the microvascular endothelium and is sealed by tight junctions (TJs) composed of claudin 5 (CLDN5) and occludin (7). Beyond the BBB lies the perivascular space (PVS), where leuko-

Related Commentary: p. 2897

Authorship note: C. Chapouly and G. R. John are co-senior authors

Conflict of interest: The authors have declared that no conflict of interest exists.

Submitted: October 18, 2016; Accepted: May 26, 2017.

Reference information: / Clin Invest. 2017;127(8):3136-3151.

https://doi.org/10.1172/JCI91301. cytes encounter CNS antigen in the context of major histocompatibility complex (MHC) (8). Distal to the PVS is a second barrier of astrocytic endfoot processes that encircle the microvascular wall, termed the glia limitans (GL) (9). Thus, access to the CNS from the vasculature requires penetration of both the $\mathrm{BBB}$ and the GL.

Previous reports identified breakdown of the endothelial BBB as a critical event in lesion pathogenesis in conditions including MS, NMO, and viral meningoencephalitis (10-12). Establishment and maintenance of the endothelial barrier depend on astrocytes (13) and pericytes $(14,15)$, and both are also linked to BBB disruption in disease $(14,16)$. Studies have identified astrocytes as important drivers of BBB breakdown in lesions: proinflammatory cytokines stimulate astrocytic production of permeability signals, which downregulate endothelial TJ proteins to induce BBB opening (1719). Importantly, however, reactive astrocytes also serve a protective role in inflammatory conditions, as their conditional elimination or suppression results in strikingly larger and more aggressive lesions $(9,20)$. Collectively, these findings suggest that both the endothelial BBB and the astrocytic GL play key protective roles in limiting parenchymal access. However, while the protective role of reactive astrocytes has been described, the mechanisms by which they control trafficking at the GL are largely uncharacterized.

In this study, we show that upon opening of endothelial TJs at the $\mathrm{BBB}$, reactive astrocytes regulate leukocyte and humoral transit by forming TJs of their own at the GL. Our data reveal that reactive mouse astrocytes in vivo, and cultured human astrocytes in vitro exposed to inflammatory stimuli, upregulate the classical TJ proteins claudin 1 (CLDN1), claudin 4 (CLDN4), and junctional adhesion molecule A (JAM-A) - the same molecules that form TJ strands in tightly sealed skin and bladder epithelia (21-23). We further show that reactive astrocytes produce structural TJ complex- 
es in response to proinflammatory signals, and use TJ proteins to corral activated $\mathrm{T}$ lymphocytes into distinct clusters. Our studies in vivo demonstrate that in models of CNS inflammation and MS, mice with conditional astrocytic deletion of the key TJ structural protein CLDN4 display exacerbated leukocyte and humoral infiltration, neuropathology, disability, and mortality.

Collectively, these findings provide evidence that the BBB and GL represent a coordinated double barrier to CNS entry, which may allow for the regulation of immune cells and factors within the PVS prior to transit into the parenchyma. Therapeutic manipulation of both barriers in combination may have greater potential than either alone to restrict CNS entry of immune components, and thus lesion pathogenesis and neurologic deficit.

\section{Results}

Induction of TJ molecules in a primary human model of reactive astrogliosis. To test whether TJs and junction-associated proteins are regulated by inflammatory factors in reactive astrocytes, we initially used an in vitro model in which primary human astrocytes are exposed to cytokines implicated in lesion pathogenesis in conditions such as MS. These included IL- $1 \beta$, IFN- $\gamma$, TGF- $\beta$, IL- 6 , and IL-17 (Figure 1, A-C, and Supplemental Figure 1, A and B; see complete unedited blots in the supplemental material; supplemental material available online with this article; https://doi.org/10.1172/ JCI91301DS1). Notably, of these factors, the transcriptional pattern induced by IL-1 $\beta$ in astrocytes is thought to resemble that of reactive astrocytes in active inflammatory lesions, including induction of adhesion molecules, cytokines, and chemokines $(19,24)$.

Importantly, immunoblotting studies revealed induction of the TJ proteins CLDN1, CLDN4, and JAM-A. CLDN1 was induced by IL- $1 \beta$, IFN- $\gamma$, and TGF- $\beta 1$, CLDN4 by IL- $1 \beta$ and TGF- $\beta 1$, and JAM-A selectively by IL-1 $\beta$ (Figure $1, A$ and B). IL-6 and IL-17 did not induce TJs or TJ-associated proteins (Supplemental Figure 1, A and $\mathrm{B})$. These findings extended preliminary data from our laboratory that CLDN1 is IL-1 $\beta$-sensitive (25). CLDN4 expression was seen 6 hours after IL-1 $\beta$ treatment, was maintained at 48 hours, and declined by 72 hours (Figure 1C). Other members of the claudin and JAM families were not detected. Claudins are structural proteins that form functional TJ strands (26), while JAM-A is a signaling protein with the capacity to modulate TJ properties, as well as binding to JAM-A ligand-expressing cells of other lineages (27). Suggesting physiologic relevance, these same 3 proteins in combination represent a pattern characteristic of tightly sealed TJs in skin and bladder epithelia (21-23). Confocal microscopy of human astrocytes confirmed CLDN1, CLDN4, and JAM-A induction and localization to the cell membrane of IL-1 $\beta$-treated human astrocytes, but not controls (Figure 1D). We also detected differential regulation by TGF- $\beta 1$ of the TJ-associated MARVEL (MAL and related proteins for vesicle trafficking and membrane link) protein tricellulin, which may alter TJ strand complexity (Figure 1, A and B), though other TJ-associated proteins, including the MARVEL protein occludin, and the adaptors cingulin and zona occludens- 1 (ZO-1), which link TJ strands to the cytoskeleton (28), were not significantly regulated by cytokines (Figure 1, A and B, and Supplemental Figure 1, A and B).

CLDN1, CLDN4, and JAM-A are coexpressed at TJs at the astrocytic GL in CNS lesions. We initially validated these data in vivo using a model induced by stereotactic cortical injection of the IL-1 vector AdIL-1, which produces asymptomatic focal inflammatory lesions that are maximal in size at 7 days postinjection (dpi) (18). Confocal imaging of sections from AdIL-1-injected C57BL/6 mice at 7 dpi confirmed expression of CLDN1, CLDN4, and JAM-A, all localizing specifically to $\mathrm{GFAP}^{+}$reactive astrocytes (Figure 1E). None of the proteins was detected in controls receiving empty vector AdDL70 control (AdCtrl). Notably, confocal imaging further revealed that while astrocytic expression of these TJ proteins was detectable in the parenchyma in lesions, immunoreactivity was strongest at astrocytic endfeet surrounding the CNS vasculature (Figure 2A and Supplemental Figure 1C). Transmission electron microscopy further revealed the presence of structures between astrocytic endfeet at the GL surrounding CNS vessels, possessing the typical ultrastructural appearance of TJ strands (Figure 2B). Moreover, immunogold labeling for CLDN4 and JAM-A confirmed that both structural and signaling components of the TJ were present in these strands (Figure $2 \mathrm{~B}$ ). In contrast, perivascular astrocytes in areas injected with empty vector control were negative for CLDN4, JAM-A, and TJ strands (Figure 2B).

To investigate relevance to disease, we studied lesions in the widely used MS model experimental autoimmune encephalomyelitis (EAE), characterized by ascending paralysis and multifocal inflammatory demyelinating spinal cord lesions (29). Immunohistochemistry of EAE lesions confirmed reactive astrocyte-specific immunoreactivity for CLDN1, CLDN4, and JAM-A (Figure 2C and Supplemental Figure 1, D and E), with all 3 proteins localizing most strongly to perivascular astrocytic endfeet (Figure 2C and Supplemental Figure 1E). Detailed analysis confirmed strong astrocytic expression of TJ proteins localizing to the GL, defined as the $\mathrm{GFAP}^{+}$ layer immediately adjacent to the laminin ${ }^{+}$astrocytic basement membrane and outside the PVS, the endothelial basement membrane, and the microvascular endothelium (Figure 2C). Expression was not detected by immunohistochemistry in other cell types in lesions, and none of the proteins was detected in the CNS in nonEAE controls. These changes accompanied loss of endothelial CLDN5 in inflammatory lesions, which we previously reported and which contributes to BBB opening (18).

Collectively, these findings show that reactive astrocytes in vitro and in vivo upregulate TJ proteins that are distinct from those found in vascular endothelium and that have a similar pattern to that seen in skin and bladder epithelia. These molecules are incorporated into complexes that possess the typical ultrastructural features of TJs. Therefore, under healthy conditions, the BBB is closed and enforced by CLDN5 and occludin. The encircling GL is open and does not express TJ proteins. In response to inflammation, particularly IL- $1 \beta$ secretion by local microglia, the BBB downregulates CLDN5 and opens, while the GL upregulates CLDN1, CLDN4, and JAM-A, and forms structural TJs (Figure 2D).

TJs in reactive astrocytes form intracellular complexes tethered to the actin cytoskeleton. To explore the structural composition of reactive astrocytic TJ complexes, we used coimmunoprecipitation to assess whether they associate with canonical intracellular TJ adaptors (Figure 3). Classical TJs, as characterized in tissues including skin, gut, and renal epithelia and vascular CNS endothelium, bind to intracellular adaptor proteins in order to anchor components of the TJ strand to the actin cytoskeleton (28). Coimmunoprecip- 


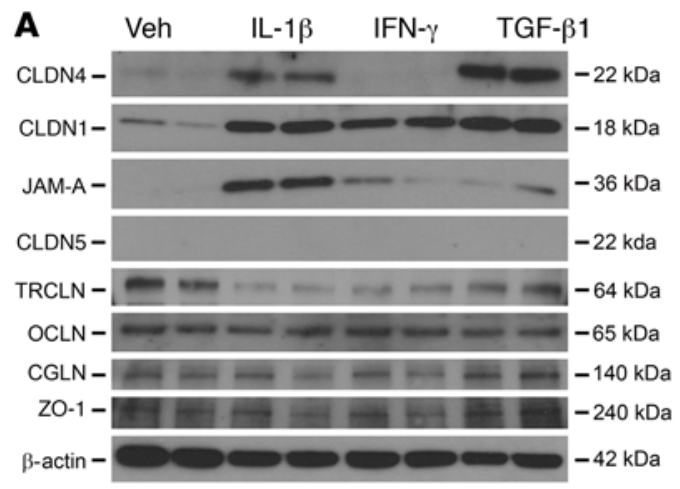

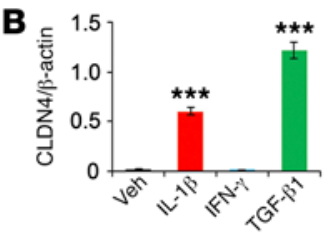
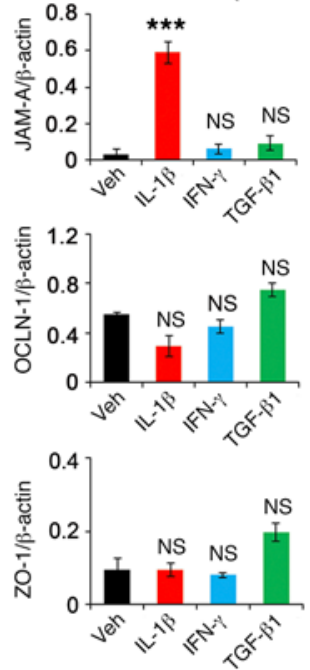
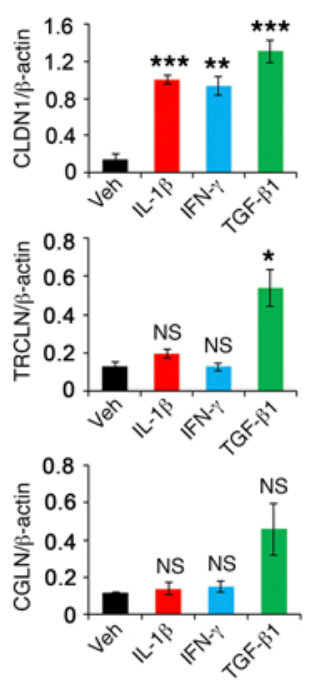

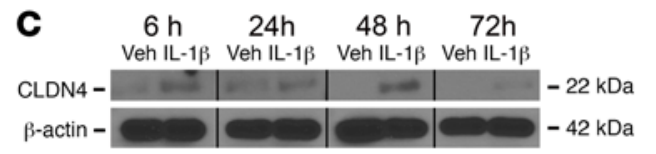

$\underline{E}$

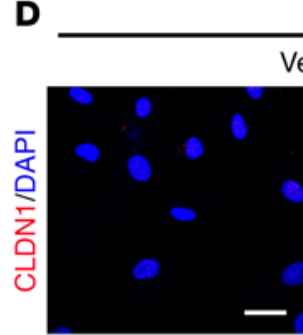

Vehicle
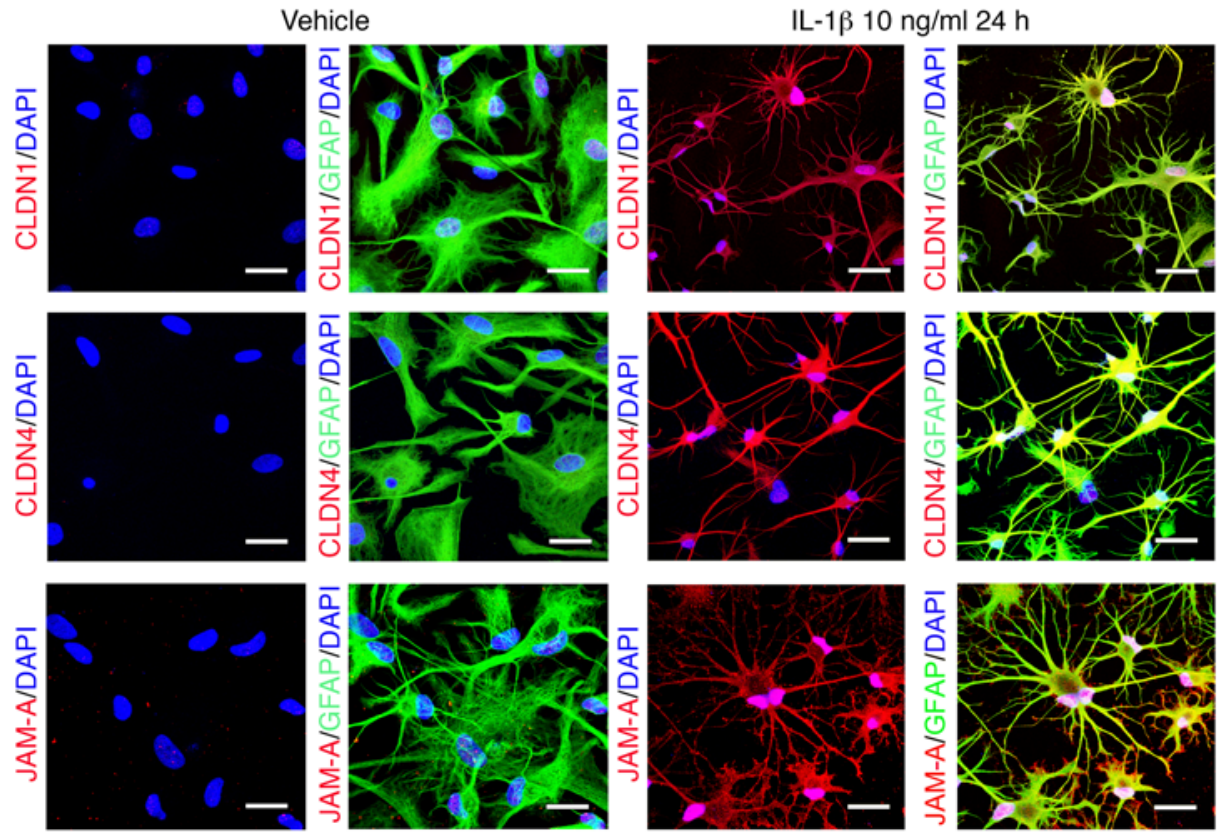
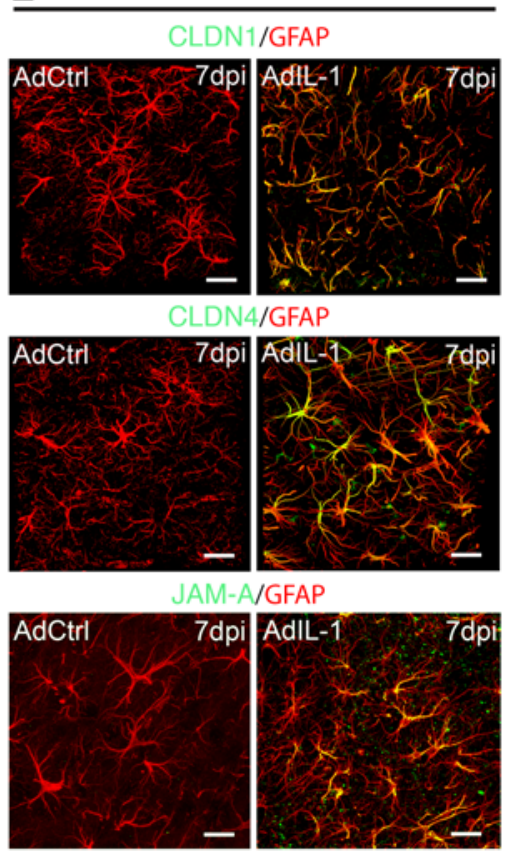

Figure 1. Reactive astrocytes upregulate CLDN1, CLDN4, and JAM-A in vitro and in vivo. (A and B) Western immunoblots (2 replicates depicted) (A) and mean values of densitometric quantification of immunoblot band intensities (from 3 biological replicates) (B) from cultured human astrocytes treated with $10 \mathrm{ng} / \mathrm{ml} \mathrm{IL-1 \beta}$, IFN- $\gamma$, or TCF- $\beta 1$ for 24 hours. CLDN1, CLDN4, and JAM-A are all induced by IL-1 $\beta$, and CLDN1 and CLDN4 are also induced by TCF- $\beta 1$. The TJ-associated protein tricellulin is induced by TCF- $\beta 1$ alone. CLDN5 is not expressed by astrocytes. See also Supplemental Figure 1, A and B. (C) Following treatment with $10 \mathrm{ng} / \mathrm{ml} \mathrm{IL-1 \beta}$, CLDN4 induction begins at 6 hours, is maintained at 24 and 48 hours, and decreases at 72 hours. (D) Immunostaining of human astrocyte cultures demonstrates that IL-1 $\beta$ induces expression of CLDN1, CLDN4, and JAM-A (red), which localize to the cell membranes of cells positive for the astrocyte marker GFAP. Scale bars: $20 \mu \mathrm{m}$. (E) In control C57BL/6 mice, cortical microinjection in vivo of adenovirus expressing IL-1 (AdIL-1), but not a control sequence, AdDL70 (AdCtrl), induces reactive astrocyte morphology and upregulation of CLDN1, CLDN4, and JAM-A at 7 days postinjection (7 dpi). Images are 3-dimensionally rendered projections. Scale bars: $40 \mu \mathrm{m}$. Data are representative of findings from 3 (A-C, and E) or more than 3 (D) biological replicates. ${ }^{*} P<0.05,{ }^{* *} P<0.01,{ }^{* *} P<0.001$. 
A
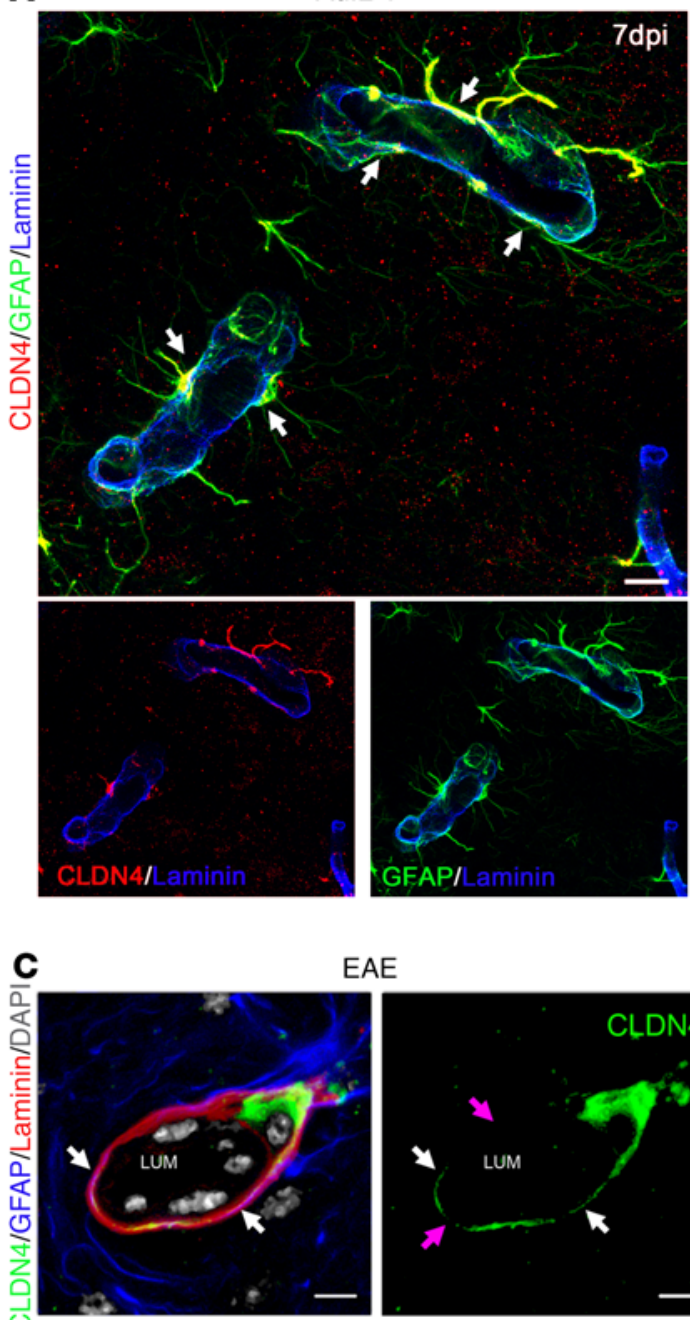

EAE

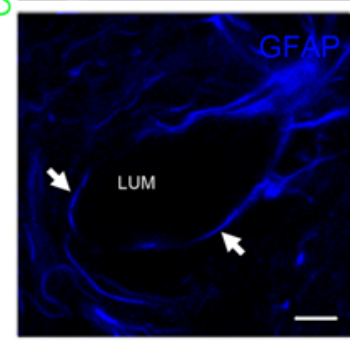

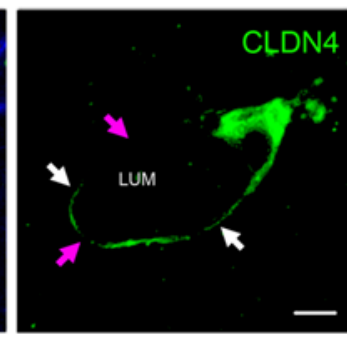

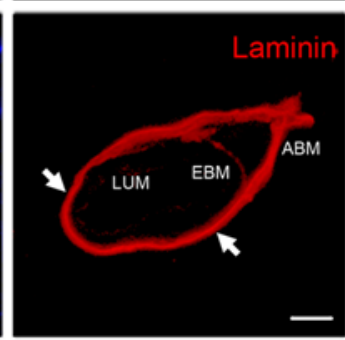

B
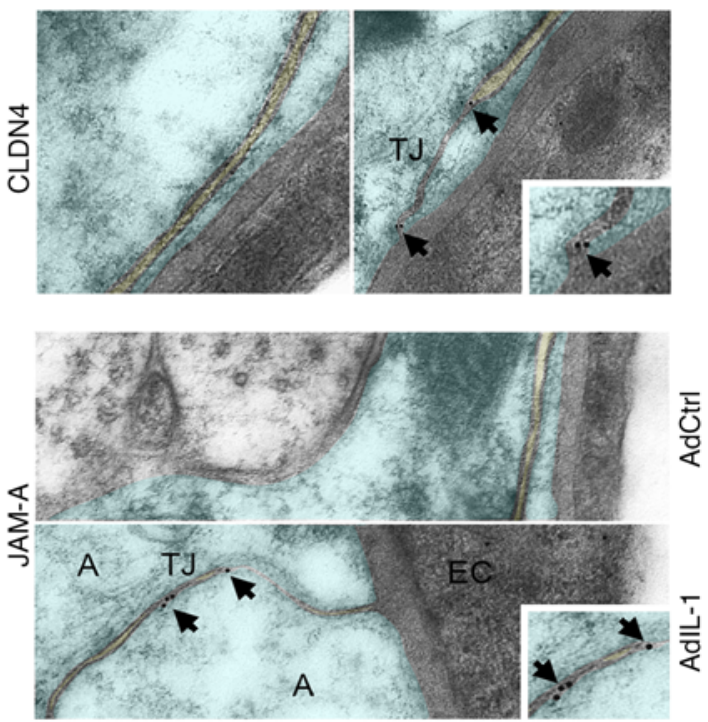

D

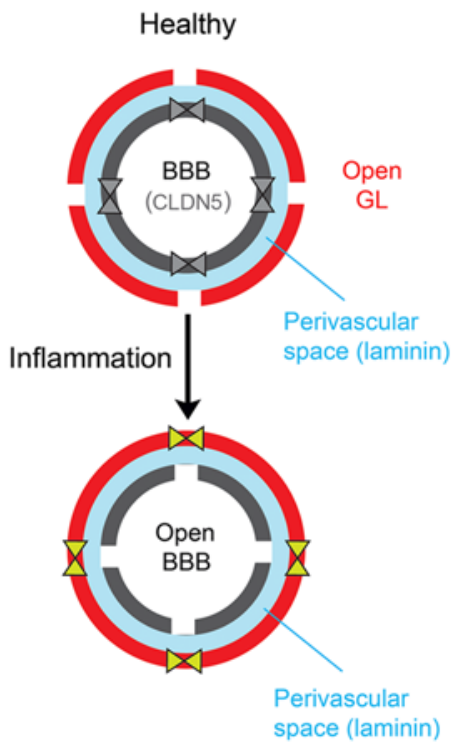

Figure 2. CLDN4 and JAM-A are expressed within TJ strands of reactive astrocytes in vivo. (A) Immunostaining within an AdIL-1 lesion at $7 \mathrm{dpi}$ for GFAP (green), the basement membrane marker pan-laminin (blue), and the astrocytic TJ protein CLDN4 (red) demonstrates CLDN4 expression at reactive astrocytic endfeet surrounding the vasculature (arrows). Scale bar: $10 \mu \mathrm{m}$. See also Supplemental Figure 1C. (B) Transmission electron microscopy of astrocytic endfeet within cortical AdIL-1 and AdCtrl injection sites demonstrates TJs in AdIL-1 lesions but not in controls. Immunogold staining shows colocalization of CLDN4 and JAM-A to the TJ structures (arrows point to gold particles). A, astrocyte; EC, endothelial cell. Original magnification, $\times 10,000$. (C) Immunostaining within an EAE lesion at 21 days for GFAP (blue), pan-laminin (red), and CLDN4 (green) demonstrates the structural organization of the reactive GL. This cross section shows basement membranes of the endothelial BBB (EBM) and astrocytic GL (ABM), demarcated by pan-laminin staining and differentiated by astrocytic endfeet, stained by GFAP and CLDN4. Leukocytes, identified in gray based on morphologic features and DAPI nuclear staining, are seen within the endothelial lumen (LUM) and PVS. White arrows highlight colocalization of CLDN4 and GFAP; pink arrows indicate areas of irregular CLDN4 staining, possibly reflecting irregularities of expression in the plane of staining or degradation in proximity to leukocytes. Scale bars: $10 \mu \mathrm{m}$. See also Supplemental Figure 1, D and E. (D) Schematic of the endothelial BBB and astrocytic GL in health and inflammatory disease. Under healthy conditions, endothelial cells express T) proteins CLDN5 and occludin (OCLN), which reinforce a closed BBB. In response to inflammation, CLDN5 and OCLN are downregulated, opening the BBB. In turn, astrocytes of the GL upregulate TJ proteins CLDN1, CLDN4, and JAM-A, closing the GL and restricting incoming leukocytes to the PVS (blue). Data are representative of findings from at least $3(\mathbf{A}-\mathbf{C})$ biological replicates. 


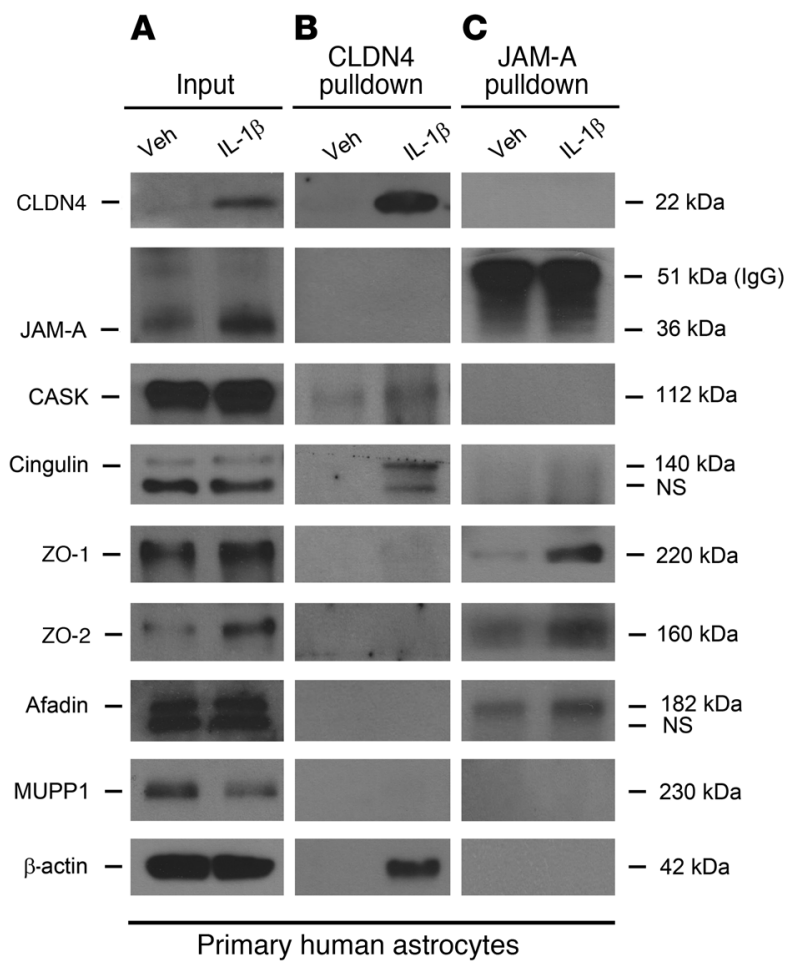

D

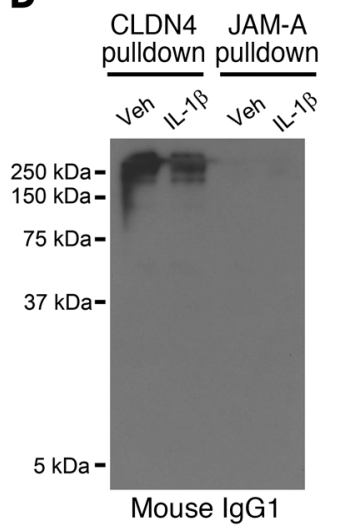

Mouse lgG1

CLDN4 JAM-A pulldown pulldown

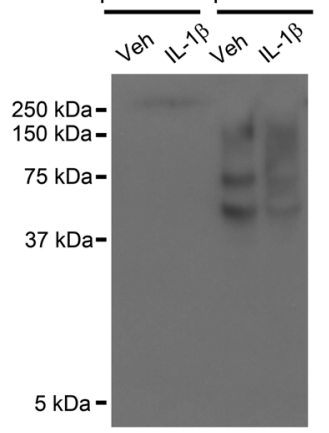

Rabbit polyclonal IgG

Primary human astrocytes
Figure 3. CLDN4 and JAM-A coimmunoprecipitate with canonical TJ complex proteins. (A-C) Immunoprecipitation of cell lysates from cultured human astrocytes under control and IL-1 $\beta$-treated conditions reveals that CLDN4 and JAM-A bind different patterns of intracellular TJ adaptor proteins. (A) Cell lysate inputs confirm that astrocytes upregulate JAM-A and CLDN4 after IL-1 $\beta$ treatment and express a variety of intracellular TJ adaptor proteins at baseline and under IL-1 $\beta$-treated conditions. (B) CLDN4 associates with cingulin, CASK, ZO-1 (the latter weakly), and $\beta$-actin. (C) JAM-A complexes with an overlapping but slightly different array of TJ-associated proteins, including ZO-1, ZO-2, afadin, and cingulin (the latter weakly). Interestingly, and compatible with previous reports (30), CLDN4 and JAM-A do not coimmunoprecipitate with each other, suggesting that the 2 proteins do not associate directly and, if connected at all, are linked via weaker bonds of intermediary proteins. (D) Nonspecific isotype control mouse IgG1 and polyclonal rabbit IgG do not show signal at the molecular weights corresponding to TJassociated proteins of interest, confirming that antibodies for $\mathrm{TJ}$ adaptor proteins are specific. Data are representative of findings from more than 3 biological replicates $(\mathbf{A}-\mathbf{D})$ itation for CLDN4 (a structural protein within the TJ strand) and JAM-A (a TJ strand-associated signaling molecule) was performed on cell lysates of human astrocytes under control and IL-1 $\beta$-treated conditions. We found that CLDN4 associated with the TJ adaptor proteins cingulin, CASK, and ZO-1 (the latter weakly), and cytoskeletal $\beta$-actin (Figure 3, A and B). JAM-A existed in complexes with an overlapping but slightly different composition, containing $\mathrm{ZO}-1, \mathrm{ZO}-2$, afadin, and cingulin (the latter weakly) (Figure 3, A and C). Interestingly, and compatible with a previous report (30), CLDN4 and JAM-A did not coimmunoprecipitate with each other, suggesting that the two, if connected within the TJ complex, do not associate directly and are more weakly linked by intermediary proteins (Figure 3, A-C). All of the tested adaptor and cytoskeletal proteins were present in total cell lysate of astrocytes under control and IL-1 $\beta$-treated conditions (Figure 3A). Nonspecific isotype control mouse IgG1 and polyclonal rabbit IgG did not show signal at the molecular weights of interest, confirming that antibodies for $\mathrm{TJ}$ adaptor proteins were specific (Figure 3D). These data suggest that in response to inflammatory stimuli, astrocytes produce structural TJ complexes resembling those found in other tissues.

Reactive astrocytes use TJ proteins to corral inflammatory cells into clusters in an in vitro model. Preparatory to functional studies in vivo, we tested whether human reactive astrocytes use TJ proteins to regulate inflammatory cell behaviors in vitro, using an adapted version of a recently described model in which primary astrocytes are cocultured with activated $\mathrm{CD}^{+} \mathrm{T}$ lymphocytes (31) (Figure 4 and Supplemental Figure 2). We nucleofected primary human astrocytes with
siRNA for CLDN1, CLDN4, JAM-A, or nontargeting control, then grew them to confluence and exposed them to IL-1 $\beta$ or vehicle for 24 hours, followed by washout. Primary human $\mathrm{CD}^{+} \mathrm{T}$ cells were activated with biotinylated anti-CD3 antibody and then added to the astrocytes. Resulting cocultures were left to interact for 24 hours.

Quality control studies confirmed silencing of CLDN1, CLDN4, and $J A M-A$ as efficient and specific (Supplemental Figure 2, A and B), and Image J analysis of confocal images validated even loading of $\mathrm{CD}^{+}$cells onto astrocytic monolayers (Supplemental Figure 2, $\mathrm{C}$ and $\mathrm{D}$ ). When activated human $\mathrm{CD}^{+} \mathrm{T}$ lymphocytes were added over control astrocytes, the 2 cell types intermingled randomly (Supplemental Figure 2, E and F). In contrast, when activated $\mathrm{T}$ lymphocytes were added to astrocytes that had been exposed to IL-1 $\beta$, the astrocytes formed a network of processes that surrounded and segregated $\mathrm{T}$ cells into discrete clusters that could be identified and counted (Figure 4A). Notably, these thin, elongated astrocytic processes resembled morphology typical of reactive astrocytes in vivo (ref. 32 and Figure 4A). Importantly, however, when activated $\mathrm{T}$ lymphocytes were seeded onto cultures of IL-1 $\beta$-treated astrocytes deficient in CLDN1, CLDN4, or JAM-A, a majority of the inflammatory cells remained randomly intermingled with the astrocytes (Figure 4, B-D), and the number of clusters and leukocyte density within clusters were both reduced significantly (Figure 4, E and F). Interestingly, we found that corralling of $\mathrm{T}$ cells into clusters requires $\mathrm{T}$ cell activation, as naive $\mathrm{T}$ cells do not cluster on IL-1 $\beta$-treated astrocytes (Supplemental Figure 2F). Therefore, both astrocytes and $\mathrm{T}$ cells must be primed for cluster formation. 
A

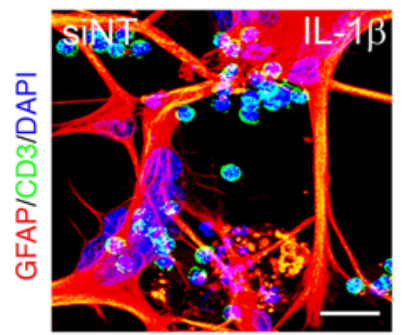

B
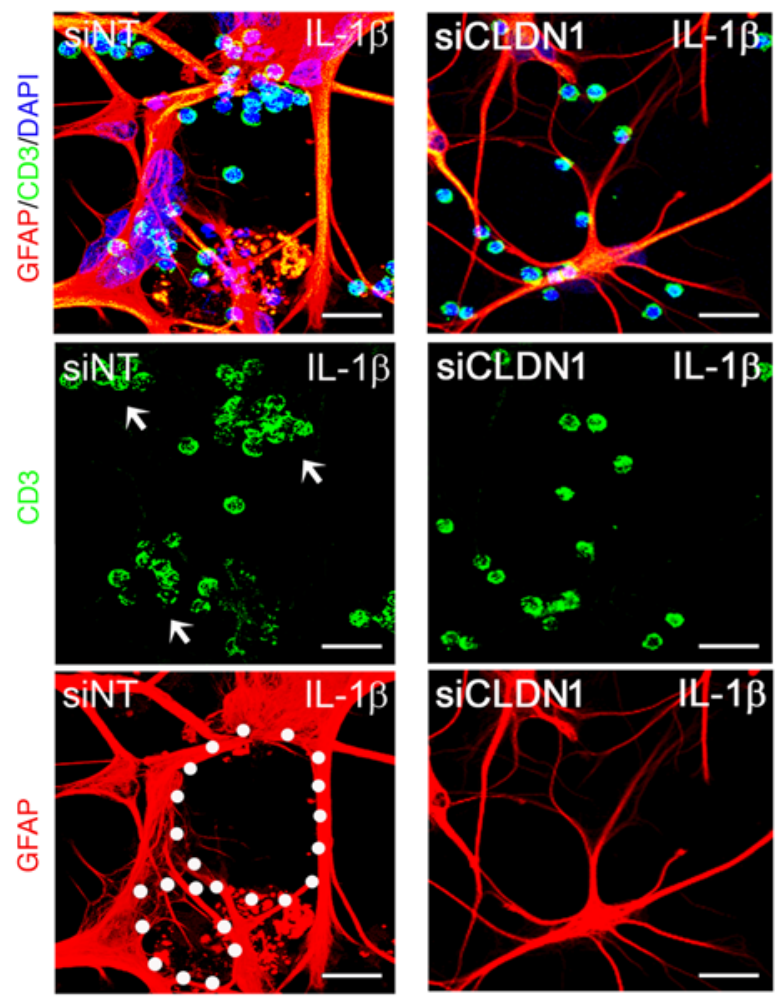

C
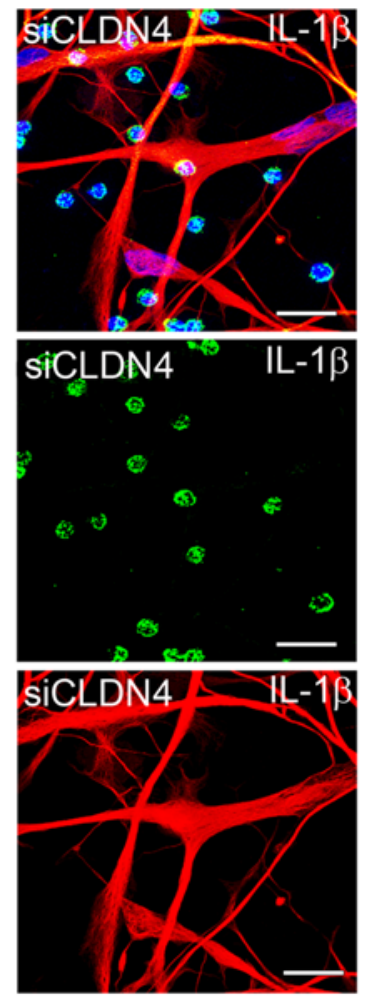

D
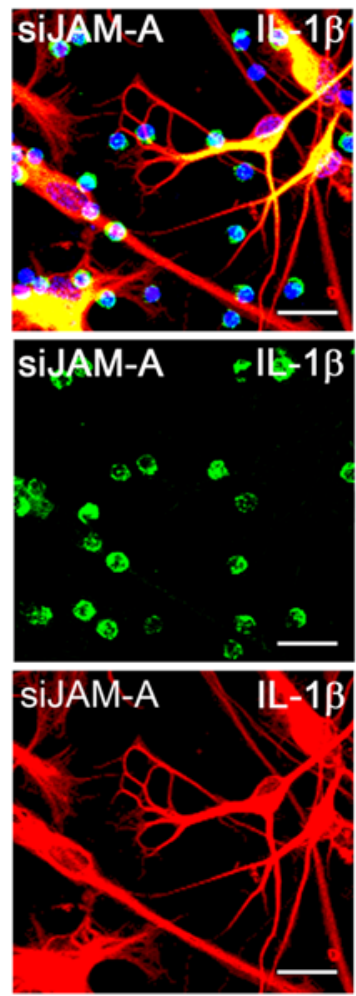

E

CD3 particle cluster outlines/

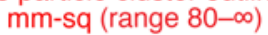
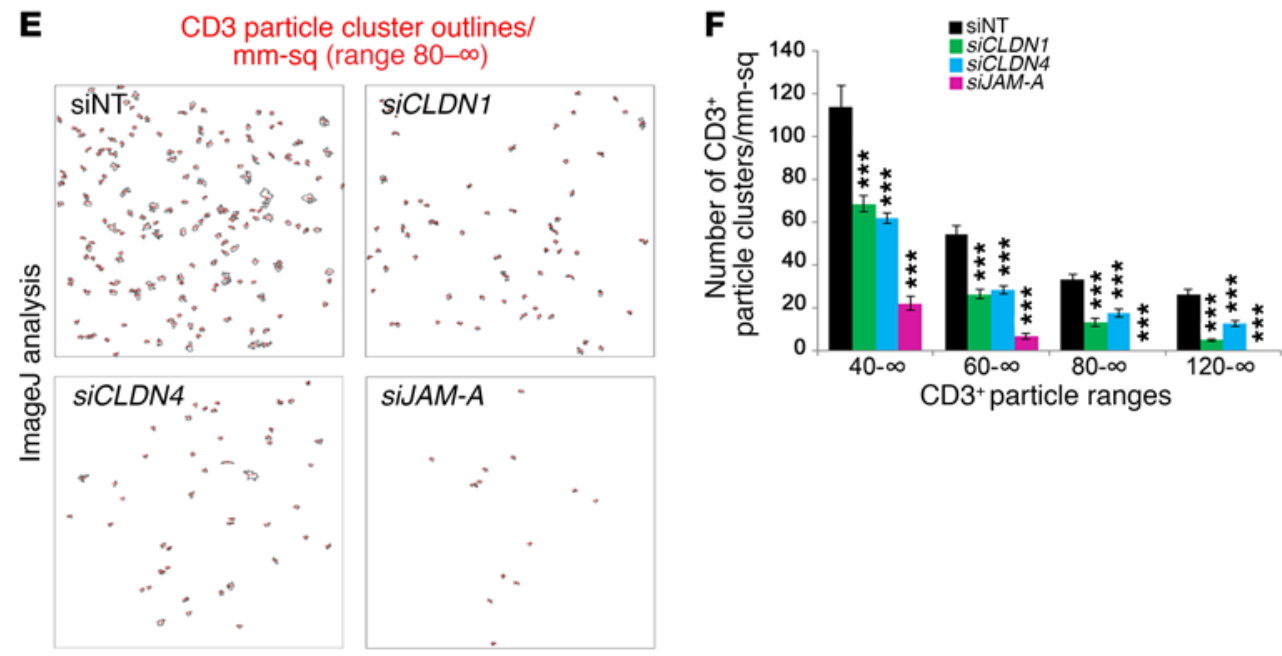

Figure 4. Silencing of astrocytic CLDN1, CLDN4, and JAM-A in vitro disrupts lymphocyte clustering. (A-D) Primary human astrocytes were nucleofected with siRNA for CLDN1, CLDN4, JAM-A, or nontargeting (NT) control, then exposed to $10 \mathrm{ng} / \mathrm{ml}$ IL-1 $\beta$ (see also Supplemental Figure $2 \mathrm{~A}$ ). After $24 \mathrm{hours,}$ astrocytes were washed and CD3+ T lymphocytes added for coculture for 24-48 hours. Scale bars: $75 \mu \mathrm{m}$. (A) Under control conditions, astrocytes extend interconnected processes that surround and corral lymphocytes into clusters (white arrows point to cell clusters, white dots outline interconnected astrocytic processes). (B-D) In cultures with silenced CLDN1, CLDN4, or JAM-A, there are fewer interconnected processes, and lymphocytes are distributed more diffusely throughout the coculture. (E and $\mathbf{F}$ ) Compared with siNT controls, there is a significant decrease in the number of clusters containing greater than $40,60,80$, and 120 cells in siCLDN1-, siCLDN4-, and siJAM-A-treated cultures ( $n=3$ per group, $P<0.00005,1$-way ANOVA with Bonferroni correction). Data are representative of 3 independent experiments in separate cultures. See also Supplemental Figure 2, B-F. ${ }^{* *} P<0.001$.

These findings show that astrocyte cultures exposed to IL-1 $\beta$ and then cocultured with activated T lymphocytes actively surround and corral these immune cells into discrete clusters. Importantly, the astrocytic TJ proteins CLDN1, CLDN4, and JAM-A each contribute to barrier formation in this model.
Astrocyte-specific Cldn4 inactivation exacerbates inflammatory lesion size in vivo. To test whether astrocytic TJs limit inflammatory lesion pathogenesis in vivo, we conditionally disrupted Cldn4 expression in reactive astrocytes, and examined the consequences in 2 inflammatory CNS models (Figure 5 and Supplemental Figure 3). 
A
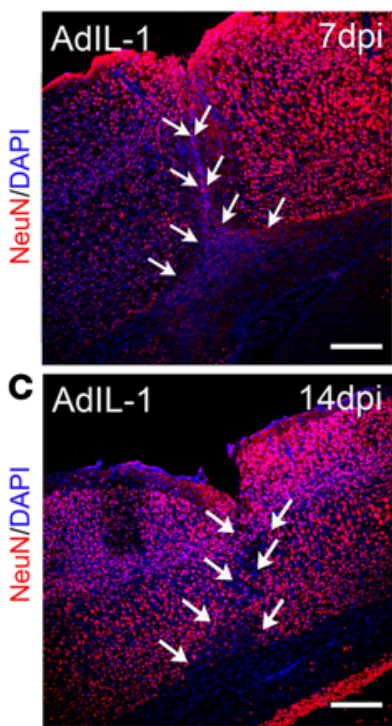

E

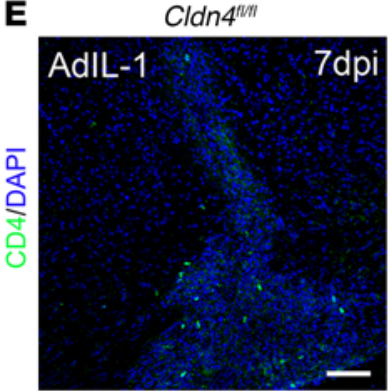

G
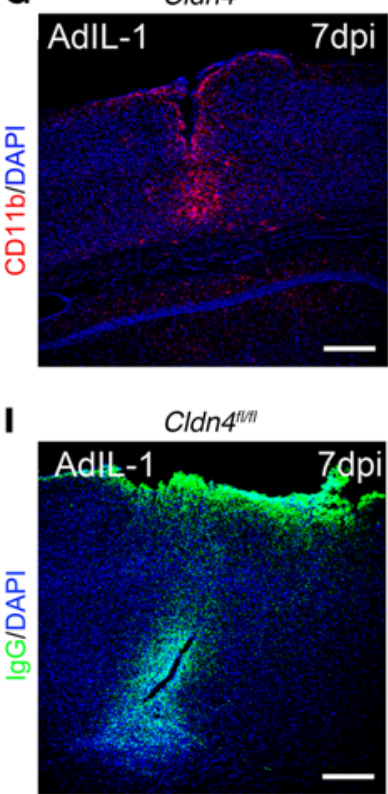

mGfap-Cre Cldn4 $4^{\text {nut }}$
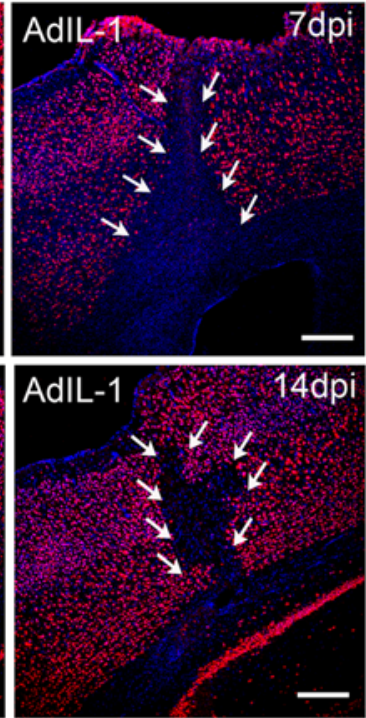

mGfap-Cre Cldn $4^{\text {InI }}$

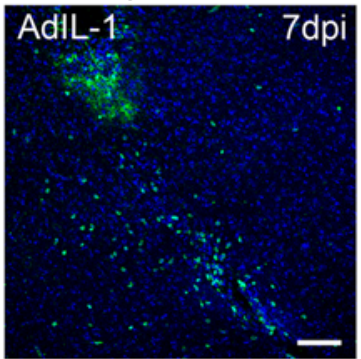

mGfap-Cre Cldn $4^{\text {IIII }}$

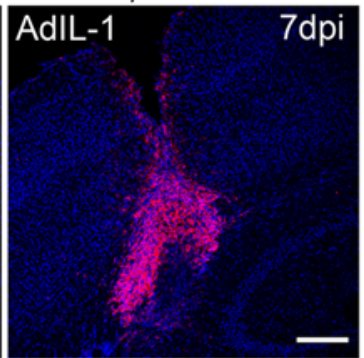

mGfap-Cre Cldn $4^{\text {IIII }}$

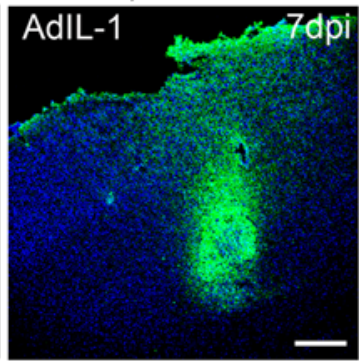

B

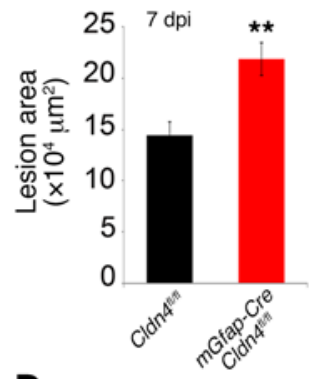

D

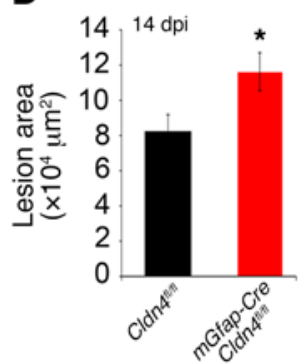

F

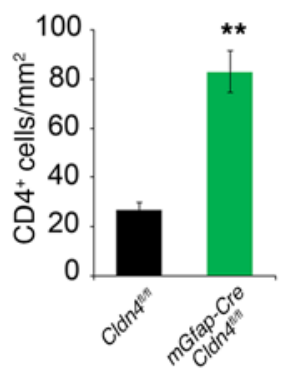

H

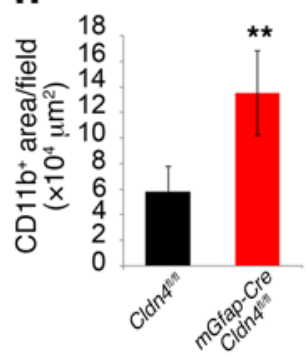

J

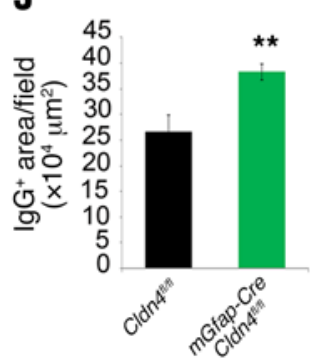

Figure 5. Conditional astrocyte Cldn4 inactivation exacerbates the size of inflammatory CNS lesions. Cortical AdIL-1 microinjection produces asymptomatic inflammatory lesions characterized by leukocyte

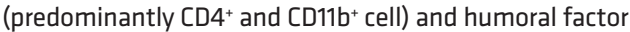
parenchymal entry, accompanied by reactive astrogliosis and neuronal death. Lesion pathogenesis peaks at $7 \mathrm{dpi}$ and is resolving by $14 \mathrm{dpi}$. (A-D) Compared with controls, Cldn4 CKO mice display increased AdIL-1 lesion size, as measured by the area of neuronal cell death (NeuN loss), at $7 \mathrm{dpi}$ and $14 \mathrm{dpi}$ (7 dpi: $n=12 \mathrm{CKO}, n=15 \mathrm{WT}$, $P<0.005 ; 14$ dpi: $n=5$ CKO, $n=8$ WT, $P<0.05$, 2-tailed $t$ test). Scale bars: $300 \mu \mathrm{m}$. (E-H) At $7 \mathrm{dpi}$, lesions of Cldn4 CKO mice have increased numbers of CD4+ lymphocytes ( $n=10 \mathrm{CKO}, n=10 \mathrm{WT}, P<0.01$, 2-tailed $t$ test) ( $E$ and $\mathbf{F}$ ) and level of CD11b staining ( $n=6$ CKO, $n=9$ WT, $P<0.005$, 2-tailed $t$ test) ( $\mathbf{G}$ and $\mathbf{H}$ ). (I and J) At $7 \mathrm{dpi}$, lesions of Cldn4 CKO mice show increased areas of IgG entry ( $n=8 \mathrm{KO}, n=11 \mathrm{WT}, P<0.01$, 2-tailed $t$ test). See also Supplemental Figure 3, A-C. ${ }^{*} P<0.05$, ${ }^{* *} P<0.01$

displayed no spontaneous pathology, and had an intact BBB (Supplemental Figure 3, A and B).

Initially, we compared responses of conditional Cldn 4 knockout (CKO) mice and controls to cortical injection of AdIL-1 versus AdCtrl, measuring the maximal area of leukocyte parenchymal entry over time, assessed by total numbers of $\mathrm{CD} 45^{+}$leukocytes and $\mathrm{CD} 4^{+} \mathrm{T}$ helper lymphocytes, and the area of $\mathrm{CD}_{11} \mathrm{~b}^{+}$macrophage/ microglial reactivity. We also measured the maximal area of humoral factor infiltration into the CNS, in terms of parenchymal entry of serum proteins with proinflammatory roles, notably fibrinogen and $\operatorname{IgG}\left(35^{-38)}\right.$. We then compared these data with the maximal area of neuronal cell death (NeuN loss) in lesions over time.

Importantly, these studies demonstrated that lesion formation in mGfap-Cre Cldn $4^{f / f l}$ mice was strongly exacerbated compared with that in littermate controls. Confirming efficacy and specificity of inactivation, AdIL-1-induced lesions in $m G f a p$-Cre Cldn $4^{f / f l}$ mice were negative for CLDN4, whereas JAM-A was induced normally (Supplemental Figure 3C). In controls, AdIL-1 induced the formation of a focal inflammatory lesion that was maximal in size at $7 \mathrm{dpi}$ and in which inflammation and reactive astrogliosis resolved by $14 \mathrm{dpi}$ (16). Notably, however, lesion size in experimental $m G$ fap-Cre Cldn $4^{f / f l}$ mice, as measured by neuronal cell death or loss of NeuN immunoreactivity, was much larger than that in controls at $7 \mathrm{dpi}$ (Figure 5, A and

We focused on CLDN4 because it represents a paradigmatic structural (as opposed to signaling) component of TJ strands. We crossed a floxed Cldn4 allele (33) with mGfap-Cre (34), to generate experimental $m G f a p$-Cre Cldn $4^{f l / f l}$ mice and 3 control genotypes ( $m G f a p$ Cre Cldn $4^{f /+}$ and non-Cre Cldn $4^{f / f l}$ and Cldn $\left.4^{f /+}\right)$. mGfap-Cre Cldn $4^{f / f l}$ mice were born in expected ratios, grew normally to adulthood,
B), and this difference persisted at 14 dpi (Figure 5, C and D). Moreover, while lesions in controls contained $\mathrm{CD} 4^{+}, \mathrm{CD} 11 \mathrm{~b}^{+}$, and $\mathrm{CD} 45^{+}$leukocytes, lesions in the CKO mice contained significantly exacerbated numbers and densities of all of these leukocyte subsets (Figure 5, E-H, and Supplemental Figure 3D). There were also large increases in the areas of cortical $\operatorname{IgG}$ and fibrinogen 


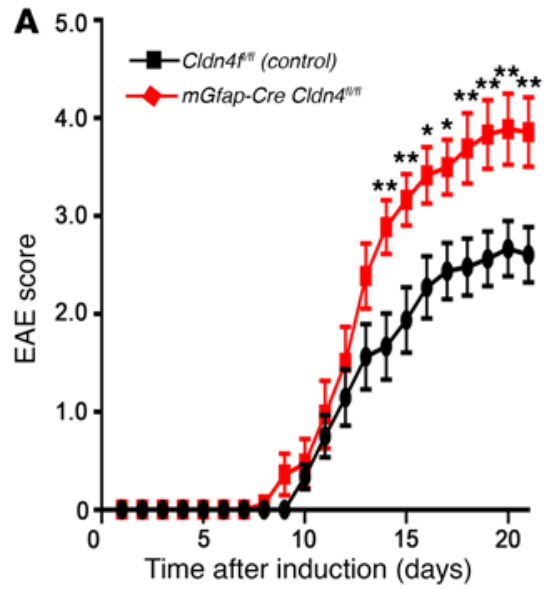

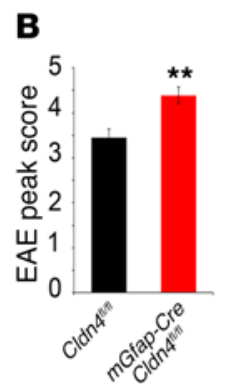
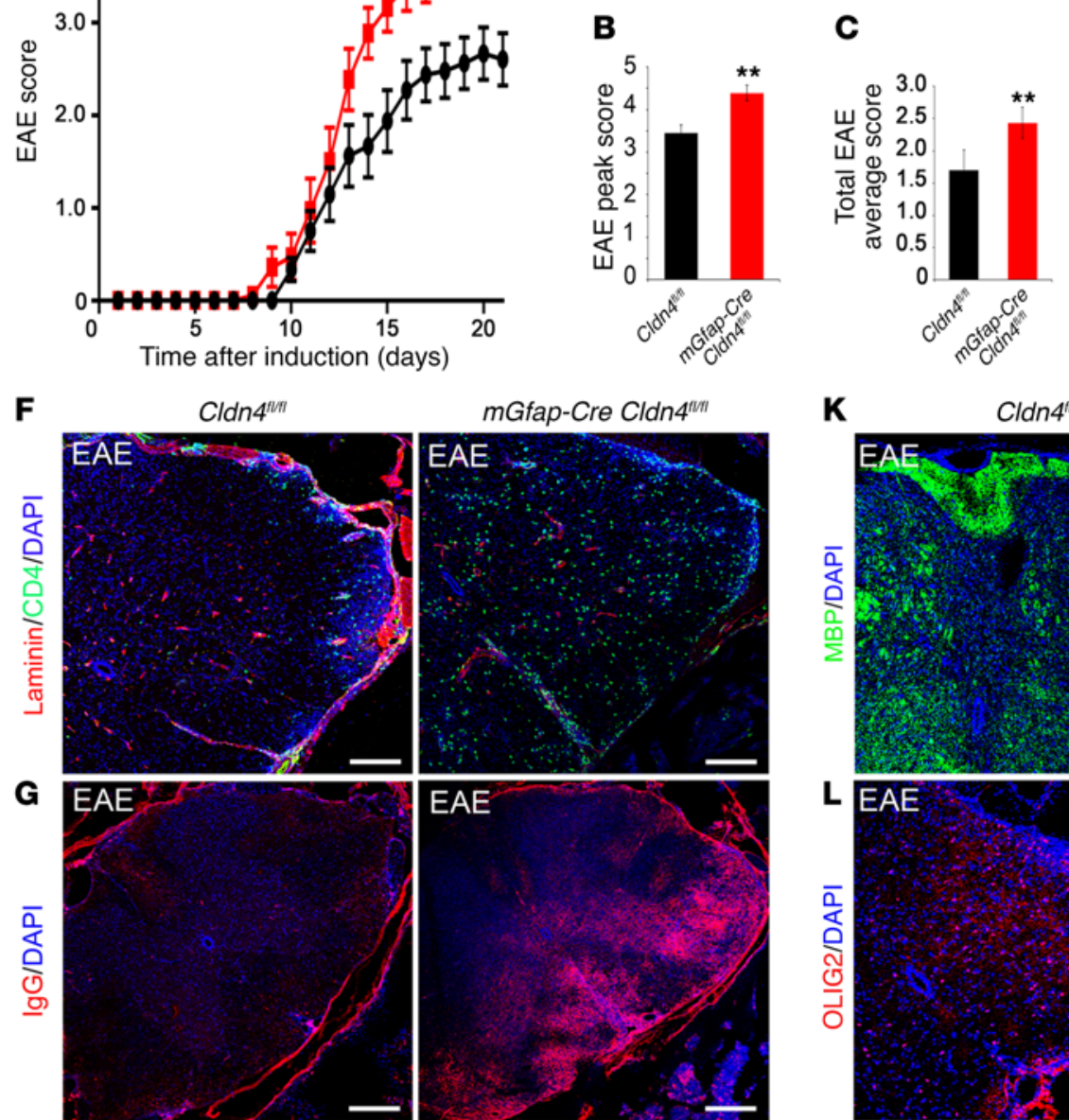

H

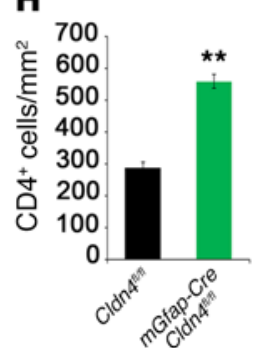

mGfap-Cre Cldn $4^{n ! n}$
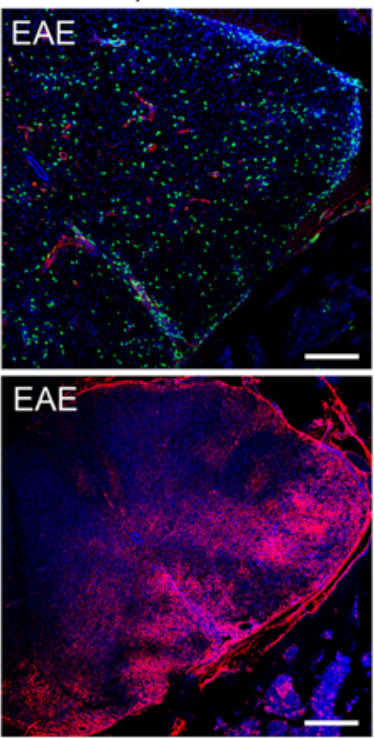

I

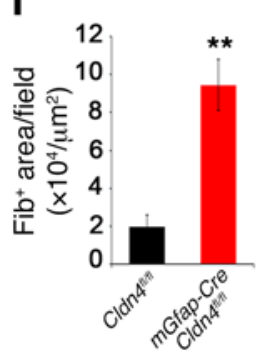

J

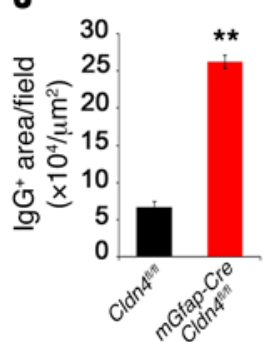

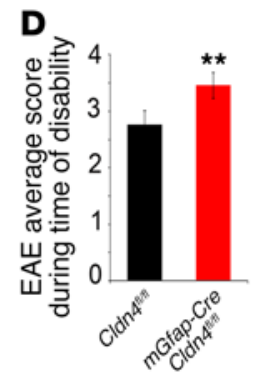

E

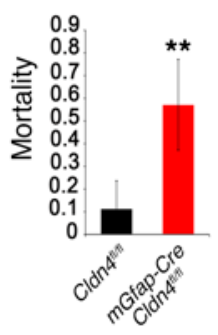

K
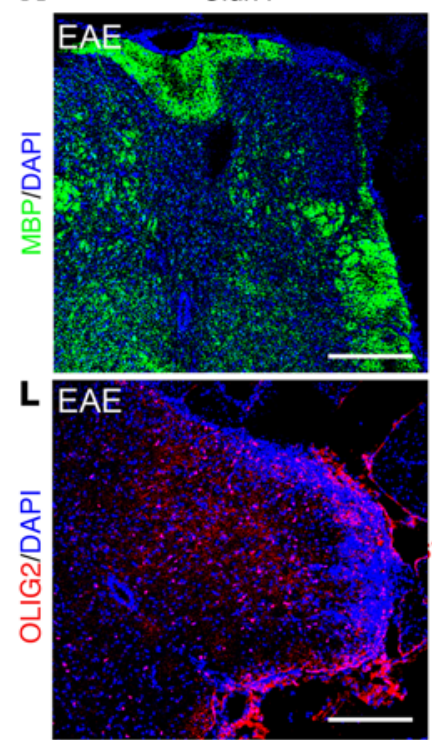

M

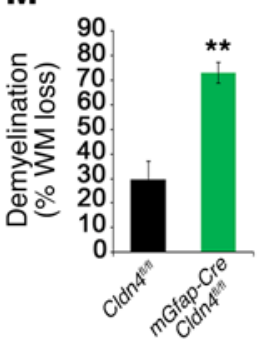

$\mathbf{N}$

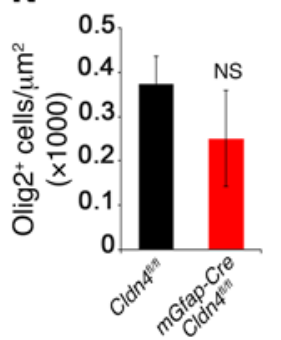

Figure 6. Clinical disability and mortality in EAE are more severe in Cldn4 CKO mice than controls. (A) Experimental Cldn4 CKO and control mice induced with EAE were scored daily on a standard 5-point scale (29). Disability scores are significantly more severe for CKO mice at days $14-21 ;{ }^{*} P<0.05$, ${ }^{* *} P<0.01$, 2-way ANOVA with Bonferroni correction. (B) Peak score during EAE is increased in Cldn4 CKO mice compared with controls (CKO $n=18$, WT $n=24, P<0.01$, 2-tailed $t$ test). (C-E) Also increased in Cldn4 CKO are average EAE disability score from days 7 to 21 (CKO $n=18$, WT $n=24, P<0.005)$ (C), average score during time of disability (CKO $n=18$, WT $n=23, P<0.05$ ) (D), and mortality or severe paralysis requiring euthanasia (score $\geq 4 ; P<0.005)(E)$. There was no difference between groups in rate of EAE induction ( $P=0.32$, data not shown). ( $F-J)$ Spinal cord EAE lesions harvested at 21 dpi or at the time of euthanasia demonstrate increased CD4 $4^{+}$cell infiltration (CKO $n=3$, WT $n=6, P<0.01$, 2-tailed $t$ test) (F and $\mathbf{H}$ ) and increased fibrinogen (CKO $n=4, W T n=4, P<0.05$ ) and lgG entry (CKO $n=4$, WT $n=3, P<0.005$ ) (C, I, and J) in Cldn4 CKO mice compared with controls. Data for CD4 ${ }^{+}$cells were confirmed using flow cytometry (Supplemental Figure 4, A and B) with no difference in counts from the spleen (Supplemental Figure 4, C and D). Infiltrating inflammatory cells in Cldn4 CKO mice showed more parenchymal access past the glia limitans superficialis and perivascular spaces compared with controls (Supplemental Figure 4, E and F). (K and $\mathbf{M}$ ) Demyelination in EAE lesions, as measured by loss of myelin basic protein (MBP), which represents the percentage of white matter loss (\% WM loss) within the dorsolateral (corticospinal motor) tracts, is strikingly increased in Cldn4 CKO mice compared with controls (CKO $n=4, W T n=4, P<0.005)$. ( $L$ and $\mathbf{N}$ ) Oligodendrocyte numbers within EAE lesions are not significantly different between groups (CKO $n=3$, WT $n=3, P=0.25)$. Scale bars: $300 \mu \mathrm{m}$ ( $\mathbf{F}$ and $\mathbf{~}), 500 \mu \mathrm{m}$ ( $\mathbf{K}$ and L). See also Supplemental Figure 4, H-K. ${ }^{*} P<0.05$, ${ }^{* *} P<0.01$. 
A

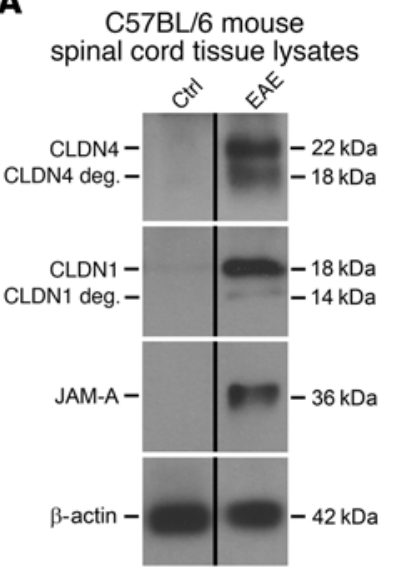

B
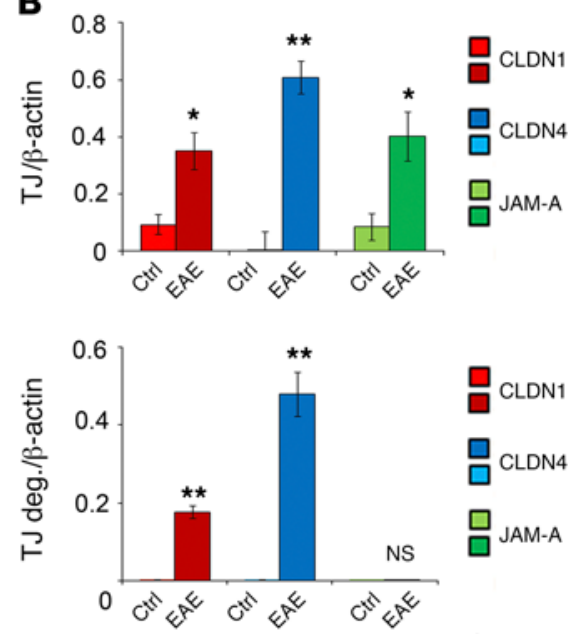

C
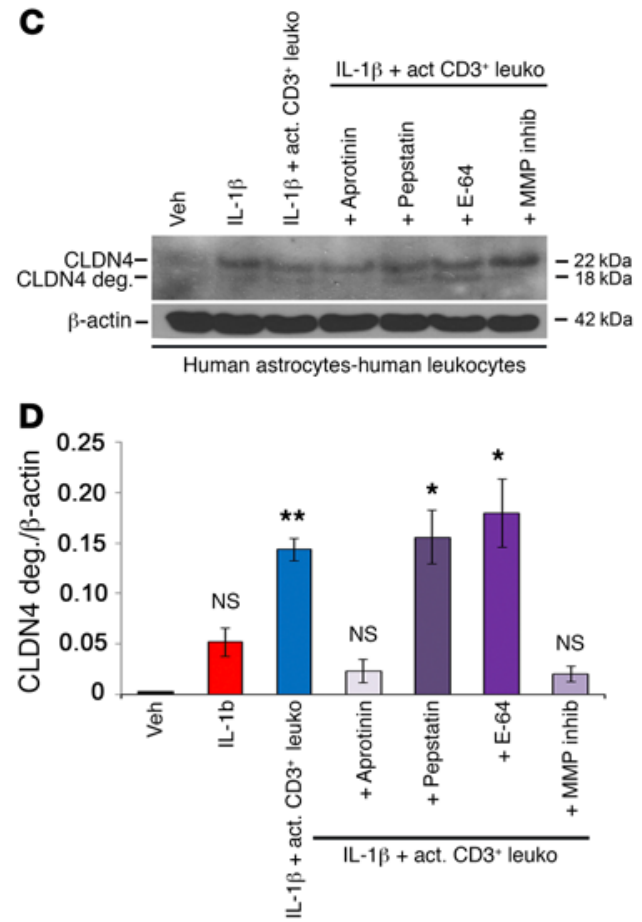

G
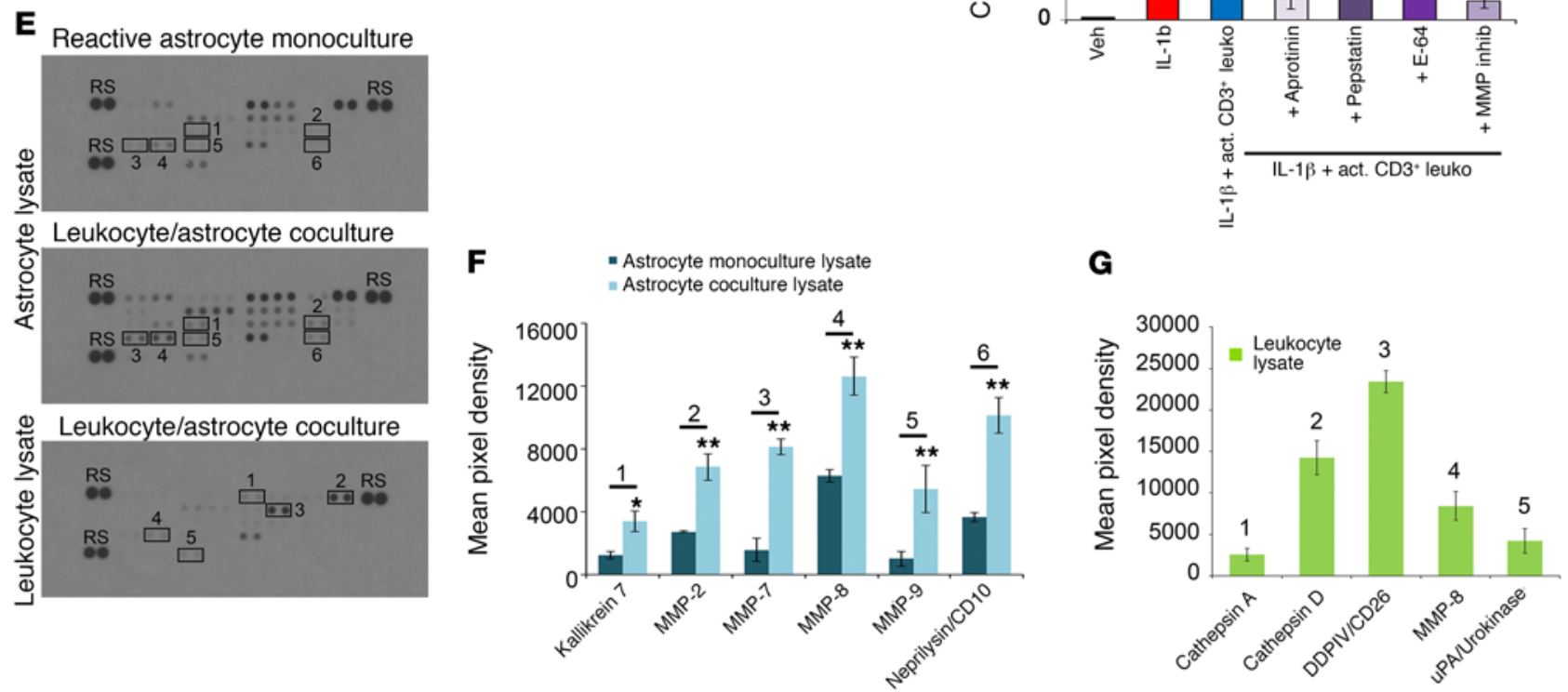

Figure 7. Astrocytic CLDN4 is degraded in EAE lesions and in coculture with activated CD3+ lymphocytes in vitro. (A and B) Immunoblotting (A) and densitometric quantification (B) of spinal cord lysates from C57BL/6 mice with EAE (score 2-3, from 18-21 days) and age- and sex-matched controls demonstrate induction of CLDN1, CLND4, and JAM-A in EAE (A and B, upper panel), along with degradation products of CLDN1 and CLDN4 (14 kDa and $18 \mathrm{kDa})$, but not JAM-A (A and B, lower panel). (C and $\mathbf{D})$ Immunoblotting and densitometry of cocultures of reactive human astrocytes (IL-1 $\beta$-treated followed by washout) with activated $\mathrm{CD}^{+}$lymphocytes. Coculture leads to degradation of astrocytic CLDN4 by 24 hours, and CLDN4 degradation is blocked by specific protease inhibitors, including the serine protease inhibitor aprotinin, and by MMP inhibitor-2. In contrast, degradation is not blocked by the cysteine protease inhibitor E-64, or the aspartic protease inhibitor pepstatin. These studies collectively suggest combinations of kallikrein and urokinase (substrates of aprotinin) and MMP-1, -3, -7, and -9 (substrates of MMP inhibitor-2) as potentially responsible for CLDN1 and CLDN4 digestion (see also Supplemental Figure 5, A-C). (E-C)

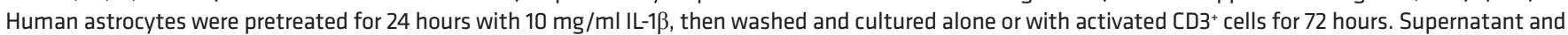
cell lysates of isolated astrocytes or leukocytes were then harvested and applied to protease arrays (3 biological replicates of each condition) (E and $\mathbf{F})$. Astrocyte lysates from coculture with $\mathrm{CD}^{+}$cells showed upregulation of kallikrein 7, MMP-2, -7, -8 , and -9 , and CD10 compared with lysate from monoculture ( $n=3$ each group, 2-tailed $t$ test, $P<0.05$ ). (E and $\mathbf{C}) \mathrm{CD3}^{+}$cell lysates from coculture demonstrated strongest expression of cathepsins $A$ and $D, D D P I V, M M P-8$, and uPA. See also Supplemental Figure 5, D and E. Data in A-G are representative of findings from 3 or more biological replicates. ${ }^{*} P<0.05,{ }^{* *} P<0.01$.

infiltration seen in response to AdIL-1 in $m$ Gfap-Cre Cldn $4^{\text {f/fl}}$ mice at 7 dpi (Figure 5, I and J, and Supplemental Figure 3, E and F). Interestingly, GFAP expression, a marker of reactive astrocytosis, was not significantly different between groups, indicating that AdIL-1 injections activated astrogliosis to a similar extent (Supplemental Figure 3G).

Mice with astrocytic Cldn4 inactivation display exacerbated disability in a model of MS. To examine the impact of these findings on disease severity, we investigated the phenotype of the MS model EAE in $m$ Gfap-Cre Cldn $4^{A / f l}$ mice and Cldn $4^{A / f}$ controls (Figure 6 and Supplemental Figure 4). Mice (10- to 12-week-old females, $n=18 \mathrm{CKO}$, $n=24$ control) were sensitized with the encephalitogenic myelin peptide $\mathrm{MOG}_{35-55}$, and clinical deficit was evaluated using a widely accepted 5-point paradigm from day 7 until the end of the experiment at day 21 after sensitization (29).

Critically, these studies revealed that the clinical course and pathology of EAE were strongly exacerbated in $m G f a p-C r e ~ C l d n 4^{f / f l}$ mice. In controls, neurologic deficit was observed from day 10, and 
increased in severity until day 20, when clinical score stabilized at a mean of 2.6, representing hind limb weakness and unsteady gait (Figure 6A). In contrast, the onset of clinical signs in $m G f a p$ Cre Cldn $4^{f / f l}$ mice was first seen 2 days earlier, and the clinical course was much more severe. In $m G f a p$-Cre Cldn $4^{f / f l}$ mice, disease reached a plateau at day 18 at a mean of 3.8, indicating hind limb paralysis and forelimb involvement, a severe clinical phenotype, and this divergence in scores reached significance from day 14 to day 21 after sensitization (Figure 6A). The peak EAE score, total average score, and score during the time of disability were all exacerbated in $m$ Gfap-Cre Cldn $4^{f / f l}$ mice (Figure 6, B-D). These changes translated to marked differences in survival. Mortality or severe paralysis requiring euthanasia (score $\geq 4$ ) occurred in $57 \%$ of $m G f a p$ Cre Cldn $4^{f / f l}$ mice, but in only $11 \%$ of $C l d n 4^{f / f l}$ littermate controls (Figure 6E).

Neuropathology in the $m G f a p$-Cre Cldn $4^{f / f l}$ cohort was also strongly exacerbated (Figure 6, F-N). Typical pathology of EAE was observed in spinal cord white matter of controls at 21 days, including inflammatory leukocyte and serum protein infiltration (Figure 6, F-J) and multifocal demyelination (Figure 6, K-N, and ref. 29). Reactive astrocytes in EAE lesions in controls expressed CLDN4, CLDN1, and JAM-A, with immunoreactivity strongest at the GL (Supplemental Figure 1, D and E). In contrast, lesions in mGfapCre Cldn $4^{f l / f l}$ mice were negative for CLDN4, whereas CLDN1 and JAM-A were induced normally (Supplemental Figure 1D). The numbers and density of inflammatory cells in lesions in mGfapCre Cldn $4^{f / f l}$ mice were strongly amplified, as measured using histopathology and confirmed using flow cytometry (Figure 6, F and H, and Supplemental Figure 4, A and B). Entry of fibrinogen and immunoglobulin was also exacerbated (Figure 6, G, I, and J). Interestingly, infiltrating inflammatory cells in mGfap-Cre Cldn $4^{f / f l}$ mice showed more parenchymal access through the glia limitans superficialis as well as past the PVS within penetrating vessels, compared with controls (Supplemental Figure 4, E and F). Importantly, these changes resulted in strikingly increased areas of demyelination in $m$ Gfap-Cre Cldn $4^{f / f l}$ spinal cords (Figure 6, K-N). These effects were CNS-specific - no differences in lymphocyte numbers were seen in peripheral lymphoid organs of $m G$ fap-Cre Cldn $4^{f l / f l}$ mice versus controls with EAE (Supplemental Figure 4, C, D, and G).

To test for qualitative rather than quantitative changes in patterns of pathology, we performed a secondary analysis on 2 outlier groups, a group of mild CKO mice $(n=3$, mean score 2.6) and a group of severe WT mice $(n=3$, mean score 3.9), euthanized at 21 dpi (Supplemental Figure 4, H-K). We found that severe WT mice had a similar degree of demyelination and numbers of $\mathrm{CD}^{+}$cells to those of representative CKO mice (Supplemental Figure 4, H and J). However, IgG infiltration into the parenchyma was significantly higher in representative CKO mice compared with both representative and severe WT mice (Supplemental Figure 4I). These data suggest that the loss of a TJ-enforced barrier at the GL in conditional knockouts allows for more diffusion of soluble IgG into the CNS than is seen even in the most aggressive EAE in WT mice.

Collectively, these results reveal that conditional loss of a key structural component of astrocytic TJs at the GL in mGfap-Cre Cldn $4^{f / f l}$ mice leads to increased leukocyte and humoral infiltration into the CNS in inflammatory lesions, and exacerbated pathology. In a model of MS, these changes result in increased paralysis and mortality. Thus, these studies identify astrocytic TJ formation as an important mechanism controlling tissue damage and clinical deficit in a model of human disease.

Activated T cells cleave astrocytic CLDN4 using serine proteases and MMPs. To understand how circulating leukocytes are able to transit the astrocytic TJ barrier in inflammatory lesions and enter the CNS, we carried out immunoblotting of spinal cord samples from WT C57BL/6 mice with EAE (score 2-3, from day 18 to day 21) and age- and sex-matched controls (Figure 7 and Supplemental Figure 5). Interestingly, these studies suggested that astrocytic claudins are degraded in inflammatory lesions in a model of MS. While CLDN1, CLDN4, and JAM-A were not detected in samples from untreated controls, they were all strongly expressed in EAE samples. Moreover, we detected apparent degradation products of both CLDN1 and CLDN4, but not JAM-A, in EAE samples (Figure 7, A and B). Degradation of CLDN4 appeared more extensive than that of CLDN1.

To analyze these changes in more detail, we used immunoblotting of cocultures of reactive human astrocytes and activated human $\mathrm{CD}^{+} \mathrm{T}$ lymphocytes, prepared as above and left in coculture for 24 hours. Notably, these experiments revealed a similar pattern of apparent CLDN4 degradation products, suggesting that CLDN4 can be digested when astrocytes interact with inflammatory leukocytes (Figure 7, C and D, and Supplemental Figure 5, $\mathrm{A}-\mathrm{C})$. These products were not detected in astrocyte monocultures or in cocultures with nonactivated $\mathrm{T}$ cells (Supplemental Figure 5, A-C). Their presence in cocultures was blocked by pretreatment with a cocktail of protease inhibitors at the beginning of the incubation period, suggesting that astrocyte-leukocyte interactions lead to the production of proteases responsible for CLDN4 degradation (Supplemental Figure 5, B and C). Similar to our findings from EAE samples, no degradation of JAM-A was observed in cocultures, such that the degradative effect appeared to be claudin-selective (data not shown).

To identify the family of proteases causing the observed effects, we tested individual components of the inhibitor cocktail. Interestingly, accumulation of degradation products was blocked by pretreatment of cocultures with the serine protease inhibitor aprotinin, and by MMP inhibitor-2, which blocks the activity of MMP-1, -3, -7, and -9 (Figure 7, C and D). Notably, MMPs have been implicated in EAE pathogenesis, are secreted by astrocytes, T lymphocytes, and myeloid cells within the PVS, and facilitate leukocyte entry past the GL into the CNS (39). In contrast, degradation product accumulation was not blocked by the cysteine protease inhibitor E-64, or the aspartic protease inhibitor pepstatin.

To further identify which specific proteases are necessary for CLDN4 degradation, we screened supernatants and cell lysates of both reactive astrocytes and activated $\mathrm{T}$ cells under conditions of degradation using profiler arrays. Of 35 different human proteases profiled in this way, positively upregulated proteases present under conditions of CLDN1 and CLDN4 degradation include MMP-2, -3, $-7,-8$, and -9, cathepsins A and D, kallikrein 7, CD10, CD26, and urokinase (Figure 7, E-G, and Supplemental Figure 5, D and E). Since both aprotinin and MMP inhibitor-2 are sufficient to block both CLDN1 and CLDN4 degradation products, these studies suggest specific combinations of kallikrein and urokinase (both substrates of aprotinin) and MMP-3, -7, and -9 (substrates of MMP inhibitor-2) as potentially responsible for CLDN1 and CLDN4 digestion. 
Collectively, these studies suggest that integral structural components of reactive astrocyte TJs are susceptible to serine protease- and MMP-mediated degradation within the PVS, notably in the presence of activated T lymphocytes. This identifies a mechanism by which activated leukocytes can eventually pass through the astrocytic barrier at the GL, and thereby enter the CNS.

\section{Discussion}

Two barriers control leukocyte and soluble factor entry from the circulation into the CNS parenchyma. In inflammatory CNS conditions such as MS and NMO, identifying key mechanisms controlling parenchymal entry of inflammatory leukocytes and humoral factors may lead to new, more effective therapies to limit relapse severity and long-term disability, and may also delay the onset of secondary progression. In healthy adults, the endothelial BBB represents a gateway to the $\mathrm{CNS}$, limiting access in part via complex TJs. In inflammatory lesions, BBB integrity is disrupted via downregulation of endothelial TJs, leading to accumulation of inflammatory leukocytes within the PVS $(18,40)$. In this study, we now report that under these conditions, reactive astrocytes form TJs of their own distal to the BBB at the GL, and in doing so take control of leukocyte and humoral factor trafficking into the parenchyma. These astrocytic TJ bonds are induced under conditions of astrocytic activation, such as in the presence of the inflammatory cytokine IL-1 $\beta$, a driver of lesion pathogenesis in MS and its model, EAE, and an established risk factor for MS (24, 41-46). IL-1 $\beta$ is expressed in acute and chronic MS and EAE lesions (47, 48) and localizes by immunohistochemistry to cells with the typical markers and morphology of macrophages and microglia (49). Reactive astrocytic TJs are composed of the structural proteins CLDN1 and CLDN4 and the signaling protein JAM-A, a pattern distinct from that of TJs in the vascular endothelium, allowing for distinct regulatory and degradation pathways (7). Interestingly, the pattern in reactive astrocytes resembles that seen in tightly sealed epithelia elsewhere in the body (21-23), suggesting that similar programs are reused to generate barriers in multiple tissues, constitutively or inducibly. Conversely, in our studies TGF- $\beta 1$ upregulated CLDN1 and CLDN4, but not JAM-A. The role of TGF- $\beta$ in CNS inflammatory disease is complex and has context-specific effects $(50,51)$, and it also contributes to glial scar formation (52), suggesting that CLDN1 and CLDN4 may also contribute to reactive glial scar formation, but that JAM-A, which potentially exerts signaling effects on incoming leukocytes, may not be recruited for structural remodeling of lesions.

CLDN4 is a key structural component of astrocytic TJs at the $G L$. Our studies here have focused primarily on CLDN4 as a structural component necessary to the integrity of astrocytic TJ complexes. Our data show that conditional inactivation of astrocytic Cldn 4 in cortical IL-1 $\beta$-induced or spinal cord EAE lesions in mice results in a strongly exacerbated inflammatory CNS phenotype. This includes strikingly enlarged inflammatory lesion size with increased entry of leukocytes and proinflammatory serum proteins, resulting in greater cell death and exacerbated tissue pathology. Our in vitro assays support the relevance of these findings to our own species, using a primary human version of a recently described rodent astrocyte-leukocyte coculture system (31). Disabling astrocytic CLDN4, CLDN1, or JAM- $A$ in this system leads to the disruption of interconnected astrocytic processes and an inability to corral leukocytes into clusters, findings compatible with a primary structural role in $\mathrm{TJ}$ barrier formation for CLDN1 and CLDN4, as reported previously in other organs and tissues. Previous studies have shown that constitutive Cldn1 inactivation in mice results in perinatal death due to loss of epidermal TJ integrity (23), while CLDN4 is also expressed in kidney and lung, and constitutive or conditional Cldn4 inactivation has been linked to urothelial hyperplasia and hydronephrosis (53), failure of chloride reabsorption (54), and susceptibility to lung injury (33). Our results thus suggest that the BBB and GL represent a coordinated double barrier to CNS entry. The presence of a dynamically sealed GL in response to inflammation may allow for containment of immune cells and soluble factors within the PVS, where regulatory signals may control subsequent steps of the immune response prior to CNS entry.

Interestingly, our in vitro data further suggest that JAM-A also plays an important role in regulating astrocytic TJ properties. However, the mechanism of action of JAM-A is likely more complex, as it has been shown both to play an initial structural role in the TJ seal, and also to transition out of the TJ to a subsequent adhesive role, binding to leukocyte JAM-A ligands and inducing diapedesis and translocation (55-58). Mechanisms underlying immune cell clustering in vitro by activated astrocytes are not yet defined, although they appear to require both structural and signaling components. Functional pathways downstream of astrocyte-lymphocyte interactions within the PVS represent an exciting area for future study. Experiments using astrocyte-specific $J A M-A$ knockout mice are under way in our laboratory, to characterize its role both in modulating the astrocytic barrier at the GL and in signaling to leukocytes within the PVS in vivo.

Functional significance of a 2-barrier system regulating access to the CNS from the vasculature. While recent studies provide extensive information on establishment of the BBB and its disruption in disease $(13,16)$, much less is known about the properties of the GL. Our data now lead us to propose that in inflammatory lesions, regulation of CNS access transitions from the endothelial BBB to the astrocytic GL. The existence of the second, astrocytic, barrier provides an additional checkpoint for leukocyte and soluble factor entry, raising important questions for future investigation. For example, does the astrocytic barrier not only control the inflammatory milieu but also actively shape the local immune response? As leukocytes accrue in the PVS between the $\mathrm{BBB}$ and this second barrier, selective docking and differential stimulation of specific leukocyte subtypes could orchestrate a diversity of local inflammatory pathways. Influences on this process could include presentation of CNS antigen in the context of MHC class II within the PVS (8), and dynamic expression of astrocytic cell adhesion molecules and signaling factors (59), as well as induction of local proteases (60-62). While previous work has revealed a critical role for local proteases, MMP-2 and MMP-9, in activating chemokine pathways within the PVS and inducing immune cells to cross the GL $(39,63)$, our data suggest that local proteases also facilitate immune cell entry by simply digesting the astrocytic TJ barrier. Furthermore, we found that unique patterns of astrocytic and lymphocytic proteases are 
induced by interactions between the 2 cell types, identifying a previously unrecognized regulatory step within the perivascular milieu. Interestingly, since the mechanisms that regulate lymphocyte containment versus degradation of astrocytic TJ proteins most likely involve different molecular mechanisms on the part of lymphocytes, they may potentially be mediated by different $\mathrm{T}$ cell subsets, such as effector and regulatory cells. Critically, these findings suggest that manipulating specific aspects of signaling between the GL and incoming leukocytes may allow for more directed therapies for immune regulation.

Broader relevance to CNS access, drug delivery, and inflammation in other organs. The striking coordinated patterns of junction reorganization we have detected at the BBB and GL have broader conceptual and translational implications. For example, it is not yet clear to what extent similar changes may occur in inflammatory lesions in other tissues throughout the body. While downregulation or reorganization of $\mathrm{TJ}$ proteins has been implicated in permeability at the $\mathrm{BBB}$ and in other tissues, for example the gut $(18,40,64)$, reports of dynamic TJ protein induction resulting in barrier formation have been rare $(65,66)$, nor is it known how such changes may affect targeting of immune responses. Our findings also have potential relevance to cancer, both in neoplasia linked to astrocytes (glioblastoma, astrocytoma) and more generally. Dynamic barrier formation in tumors occurring as a result of accompanying inflammation may have potential implications for delivery of therapeutics.

Along similar lines, and importantly, it is unknown how the changes we have detected at the GL may impact the pharmacokinetics of drugs that must enter the CNS in conditions such as MS. For successful delivery of neuroprotective and regenerative agents to the CNS, our data suggest that it may be necessary to consider passage not only across the BBB, but also through the astrocytic TJ barrier.

Therapeutic implications to limit disability in MS and NMO. In summary, our data suggest that the BBB and GL represent a coordinated double barrier to CNS entry (illustrated in Figure 2D). During inflammatory lesion pathogenesis in the CNS, astrocytes first contribute to loss of integrity at the endothelial BBB, allowing entry of leukocytes into the PVS, where they encounter CNS antigen and a proinflammatory microenvironment $(16,17$, 19). Then, astrocytes use TJs of their own to control trafficking of these cells through the GL and into the parenchyma. These findings suggest that increasing efficiency of the GL barrier, for example by inhibiting expression of proteases used to degrade it, may represent a potential approach to prevent leukocyte and humoral entry into inflammatory lesions. Moreover, manipulation of the BBB and GL in combination may have greater potential than either alone to control CNS entry of leukocytes and proinflammatory soluble factors in conditions such as MS and NMO. Identifying key mechanisms controlling leukocyte and humoral entry may lead to new therapies to effectively limit relapse severity and clinical disability in patients, and potentially also prevent the onset of secondary progression.

\section{Methods}

Cell culture: monocultures. Primary human fetal astrocyte cultures were established from 3 different brains as previously described
(67). We confirmed an identical in vitro phenotype of commercially available cells (Lonza) and cross-referenced these to previous phenotypes. To establish human $\mathrm{CD}^{+} \mathrm{T}$ lymphocyte cultures from human blood of healthy donors, density centrifugation using Ficoll-Paque PLUS (GE Healthcare) was initially performed to isolate peripheral blood mononuclear cells (PBMCs) from whole blood. Six milliliters of whole blood containing acid citrate dextrose anticoagulant (Biological Specialty Corp.) was diluted with an equal volume of HBSS (Mediatech Inc.) and carefully layered in 15-ml tubes prefilled with 4 $\mathrm{ml}$ of density gradient medium. Tubes were centrifuged 40 minutes at 900 relative centrifugal force (RCF). PBMCs were collected by transfer pipette and afterward washed 2-3 times in HBSS (Mediatech Inc.) and centrifuged 15 minutes at 250 RCF. Human $\mathrm{CD}^{+} \mathrm{T}$ lymphocytes were then enriched from PBMCs using a MACS human Pan $\mathrm{T}$ Cell Isolation Kit (Miltenyi Biotec) according to the manufacturer's instructions. After isolation, human $\mathrm{CD}^{+} \mathrm{T}$ lymphocytes were cultured in RPMI 1640 (Life Technologies), 10\% FBS (Life Technologies), $1 \mathrm{ml}$ 2-mercaptoethanol (Sigma-Aldrich), $10 \mathrm{ml}$ L-glutamine (Invitrogen), and $1 \mathrm{ml}$ penicillin/streptomycin (Invitrogen). $\mathrm{CD}^{+} \mathrm{T}$ lymphocytes were activated by coating of dishes with human antiCD3 antibody ( $7 \mu \mathrm{g} / \mathrm{ml}$ in PBS) (eBioscience) for 2 hours at $37^{\circ} \mathrm{C}$ and by addition of anti-CD28 antibody $(2 \mu \mathrm{g} / \mathrm{ml}$ ) (eBioscience) to the medium. The cells were then cultured for 3 days.

Cell culture: cocultures. Astrocytes were plated to $70 \%$ confluence and cultured in DMEM, $10 \%$ serum, for 24 hours. Astrocytes were then serum-starved for 24 hours and treated with human recombinant IL-1 $\beta$ (PeproTech) for 24 hours. Astrocyte-CD3 ${ }^{+} \mathrm{T}$ lymphocyte cocultures were prepared by addition of a suspension of $1 \times 10^{5} \mathrm{CD}^{+} \mathrm{T}$ lymphocytes to an area of $1.9 \mathrm{~cm}^{2}$ confluent activated astrocytes, and cells were cultured for 1-3 days. Cocultures grown on glass confocal plates (Mat-Tek) were fixed for 30 minutes in ice-cold, freshly depolymerized $4 \%$ paraformaldehyde. Cocultures grown in 6-well plates (Corning) were harvested for Western blotting.

Cytokines/growth factors. Human IL-1 $\beta$, IL-6, and IL-17 were purchased from PeproTech. Human IFN- $\gamma$, TNF- $\alpha$, and TGF- $\beta 1$ were from $\mathrm{R} \& D$ Systems. Based on previous studies, factors were routinely used at $10 \mathrm{ng} / \mathrm{ml}(67)$.

Drugs. Aprotinin, MMP inhibitor-2 (CAS 203915-59-7), and pepstatin A were purchased from EMD Millipore and used at $8 \mathrm{nM}, 25 \mathrm{nM}$, and $1 \mu \mathrm{M}$ respectively, per the manufacturer's recommendations and according to their known $\mathrm{EC}_{50}$ values. E-64 was purchased from SigmaAldrich and used at $10 \mu \mathrm{M}$.

Antibodies. Catalog numbers of all antibodies from their respective manufacturers are shown in parentheses. Anti-CLDN1 (374900, mouse), anti-CLDN4 (329400, mouse), anti-CLDN5 (352500, mouse), anti-cingulin (PA31654, rabbit), anti-GFAP (130300, rat), and anti-mouse IgG (A11029, goat) were from Invitrogen. Anti-ZO-1 (617300, rabbit) was from Life Technologies, and anti-ZO-2 (rabbit) was from Zymed. Invitrogen, Life Technologies, and Zymed are all divisions of Thermo Fisher Scientific. Anti-JAM-A (sc-25629, rabbit) and anti- $\beta$-actin (sc-47778, mouse) were from Santa Cruz Biotechnology. Anti-JAM-A (564908, rat) was from BD Biosciences. Anti-fibrinogen (A0080, rabbit) was from Dako. Anti-CD3 (16-0037-85), antiCD4 (14-9766-82), anti-CD11b (14-0112-82), and anti-CD45 (550539, all rat) were from eBioscience. Anti-NeuN (MAB377, mouse) and antimyelin basic protein (anti-MBP) (MAB386, rat) were from Millipore. Anti-laminin (L9393) and anti-afadin (A0349, both rabbit) were from 
Sigma-Aldrich. Anti-MUPP1 (611558, mouse) was from BD Transduction. Anti-CASK (AM20099PU-N, mouse) was from Acris.

Coimmunoprecipitation and Western blotting. For coimmunoprecipitation, CLDN4 or JAM-A proteins were immunoprecipitated with mouse anti-CLDN4 antibody (Invitrogen) or rabbit anti-JAM-A antibody (Santa Cruz Biotechnology) using a coimmunoprecipitation protocol described by Cell Signaling Technology (http://www.cell signal.com/contents/resources-protocols/immunoprecipitationprotocol-(for-analysis-by-western-immunoblotting)/ip-western? $\mathrm{Ntk}=$ Content $\& \mathrm{~N}=4294956305 \& \mathrm{Ntt}=\mathrm{immunoprecipitation} \&$ fromPage $=$ plp). SDS-PAGE and Western blotting were performed as previously reported (29). For densitometry, nonsaturated developed films were scanned using a Canon LiDE scanner (Canon USA), and mean pixel density of each band was measured using ImageJ software $(\mathrm{NIH})$. Data were standardized to actin, and fold change versus control was calculated.

siRNA. Human astrocyte cultures were nucleofected with siRNA $(2 \mu \mathrm{M}$ ) for $C L D N 1, C L D N 4$, or JAM-A (Thermo Scientific Dharmacon, siGENOME SMART pool), using an Amaxa nucleofector (program A033) with the Basic Glial Kit (Amaxa) according to the manufacturer's instructions. At 24 hours, cells were serum-starved for 24 hours, and then treated as described. Controls included nontargeting siRNA and sham control (transfection reagent alone). The extent and specificity of gene silencing were assessed by immunoblotting.

Protease arrays. Reactive astrocyte and $\mathrm{CD}^{+} \mathrm{T}$ lymphocyte cocultures were prepared as outlined above. Reactive astrocyte monocultures were prepared as a control. Supernatant from cocultures and astrocyte monocultures were collected after 3 days. Reactive astrocytes and $\mathrm{CD}^{+} \mathrm{T}$ lymphocytes were then isolated from coculture or monoculture and cell lysates harvested. Samples, including $500 \mu \mathrm{l}$ of supernatant from cocultures and monocultures, $100 \mu \mathrm{g}$ of reactive astrocyte lysates from cocultures and monocultures, and $\mathrm{CD}^{+} \mathrm{T}$ lymphocyte lysates from coculture, were all processed on human protease arrays (R\&D Systems, ARY021B) per the manufacturer's protocol in biological triplicates. Quantification of protease signal was performed by densitometry as described above for Western blots.

Mice. $m$ Gfap-Cre mice were genetically engineered in the laboratory of Michael Sofroniew (UCLA) (34). Cre expression is astrocytespecific except in areas of adult neurogenesis, where it is also observed in some neural progenitors (34). Cldn $4^{f l}$ mice were genetically engineered in the Will Rogers Institute Pulmonary Research Center at the University of Southern California (33). Experimental Cldn4 CKO (mGFAP-Cre Cldn $\left.4^{f / f t}\right)$ and control (Cldn $\left.4^{f / f f}\right)$ mice were bred crossing CKOs to controls; 50/50 proportions were born, as expected. Both $m$ GFAP-Cre and Cldn $4^{f / f}$ lines were backcrossed on a C57BL/6 background for at least 12 generations. JAM- $A^{f l}$ mice were obtained from Charles Parkos (University of Michigan, Ann Arbor, Michigan, USA) and Terence Dermody (University of Pittsburgh, Pittsburgh, Pennsylvania, USA) (55). For studies using AdIL-1, mGfap-Cre Cldn $4^{f l+}$ mice were crossed with $C l d n 4^{f l / f l}$ animals to generate mGfap-Cre $C l d n 4^{f / f l}$ mice and 3 control genotypes. mGfap-Cre JAM-A $A^{f l f l}$ mice were generated using a similar strategy. For EAE, $m$ Gfap-Cre Cldn $4^{f / f l}$ mice were crossed with $C l d n 4^{f / f l}$ animals to generate $m$ Gfap-Cre $C l d n 4^{f / f l}$ mice and $C l d n 4^{f / f l}$ controls. Genotyping primers were: mGfap-Cre forward (GfF) ACCAGCCAGCTATCAACTC, reverse (GfR) TATACGCGTGCTAGCGAAGATCTCCATCTTCCAGCAG,
$350 \mathrm{bp} ; \mathrm{Cldn}^{f l}$ forward (C4fF) CAGTAGGAAAGTTGCTGTTGAGGC, reverse (C4fR) CTCCCGTGAGACAAGAGAATGAAG, band $189 \mathrm{bp}$ (wt), $257 \mathrm{bp}$ (flox). PCR conditions for $m$ Gfap-Cre mice were: $94^{\circ} \mathrm{C}$ for 4 minutes; 35 cycles of $94^{\circ} \mathrm{C}$ for 30 seconds, $56^{\circ} \mathrm{C}$ for 30 seconds, and $72^{\circ} \mathrm{C}$ for 30 seconds; then $72^{\circ} \mathrm{C}$ for 10 minutes. PCR conditions for $\mathrm{Cldn} 4^{f l}$ mice were: $94^{\circ} \mathrm{C}$ for 2 minutes, then 35 cycles of $94^{\circ} \mathrm{C}$ for 20 seconds, $60^{\circ} \mathrm{C}$ for 30 seconds, and $72^{\circ} \mathrm{C}$ for $60 \mathrm{sec}-$ onds; then $72^{\circ} \mathrm{C}$ for 10 minutes. C57BL/ 6 mice were purchased from the Jackson Laboratory.

CNS microinjection. Mice (8-12 weeks old, at least 5 per condition per time point, on the C57BL/6 background) were anesthetized using isoflurane and placed into a stereotactic frame (Kopf). AdIL-1 or AdDL70 control (AdCtrl) (10 $\left.70^{7} \mathrm{PU}\right)$ was microinjected into the cerebral cortex at $y=1 \mathrm{~mm}$ caudal to bregma, $x=2 \mathrm{~mm}, z=1.5 \mathrm{~mm}$ as previously described (18). All studies were approved by IACUC.

$E A E$. Mice (female, $10-12$ weeks old, at least 10 per group, on the C57BL/6 background) were injected s.c. with $0.1 \mathrm{cc}$ of $\mathrm{MOG}_{35-55}$ (Hooke Laboratories) at cervical and lumbar sites followed by i.p. injection of 0.1 cc pertussis toxin on day 0 and day 1 . Mice were rated by an investigator blinded to genotype on a standard 5-point motor scale from days 7-21 after induction: 0, no symptoms; 1 , floppy tail; 2 , hind limb weakness (paraparesis); 3, hind limb paralysis (paraplegia); 4, forelimb and hind limb paralysis; 5 , death (29).

Flow cytometry. WT and KO mice were anesthetized and perfused with $5 \mathrm{ml}$ PBS $1^{\prime}$ at 19-21 days after EAE induction. Animals at peak disease for longer than 6 days were excluded from analysis. Spinal cords and spleens were collected in cold PBS and mechanically dissociated. Spleen samples were passed through a $70-\mu \mathrm{m}$ filter, then incubated in red blood cell (RBC) lysis buffer (BioLegend) for 2 minutes at room temperature and washed with PBS. Spinal cords were prepared as previously described (68). Briefly, tissues were chemically dissociated with papain and passed through a 100- $\mu \mathrm{m}$ filter, and myelin was separated using a Percoll gradient. Cell suspension was then collected, incubated in blood cell lysis buffer (RBC lysis buffer, BioLegend) for 2 minutes at room temperature, and washed with PBS. Cells from spleens and spinal cords were then incubated with the PerCP-conjugated anti-CD4 antibody (BioLegend, 100434) or PE-conjugated anti-CD45 antibody (BioLegend, 103114) in cell staining buffer (BioLegend). Cells were washed twice and resuspended in cell staining buffer and DAPI. Single stainings of tissue samples were used for both tissue types. Forward scatter and side scatter were used to gate cells excluding debris and cell aggregates, and $\mathrm{CD}^{+}$and $\mathrm{CD} 45^{+}$cells were gated on the $\mathrm{DAPI}^{+}$live cell population. Samples were analyzed using a flow cytometer (LSRFortessa, BD) at the Flow Cytometry CoRE at Mount Sinai.

Immunohistochemistry. Embedding and immunostaining were as previously described (18) unless otherwise indicated. For CLDN1, CLDN4, JAM-A, MBP, IgG, fibrinogen, and Olig2, before blocking, sections were soaked in citrate $\left(\mathrm{pH} 7.5 ; 100^{\circ} \mathrm{C}\right)$. For CLDN5, before blocking, sections were soaked in EDTA $\left(\mathrm{pH} 6.0 ; 100^{\circ} \mathrm{C}\right)$. For laminin, $\mathrm{CD} 4, \mathrm{CD} 11 \mathrm{~b}$, and CD45, sections were treated with $0.5 \mathrm{mg} / \mathrm{ml}$ protease XIV (Sigma-Aldrich) at $37^{\circ} \mathrm{C}$ for 5 minutes. Primary antibodies were used at 1:100 except CLDN4 (1:50) and fibrinogen (1:1,000). Samples were examined using a Leica Microsystems confocal microscope, and stacks were collected with $z$ of $1 \mu \mathrm{m}$.

Electron microscopy. Mice were perfused transcardially with ice-cold $2 \%$ paraformaldehyde and $2 \%$ glutaraldehyde in $0.1 \mathrm{M}$ 
phosphate buffer (PB, pH 7.2). The brain was postfixed for 6 hours in the same fixative, washed in $\mathrm{PB}$, then sectioned at $400 \mu \mathrm{m}$ thickness on a vibratome (Leica) for electron microscopic embedding. Freeze substitution and low-temperature embedding of the 400$\mu \mathrm{m}$ sections were performed as previously described (69). Ultrathin sections were cut into 70-nm-thick sections with a Diatome diamond knife (Electron Microscopy Sciences) and mounted on each grid (Electron Microscopy Sciences). Postembedding immunogold labeling was carried out as described previously with some modifications (69). Sections were treated with $1.0 \%$ sodium borohydride and $50 \mathrm{mM}$ glycine to remove excess aldehydes, then rinsed thoroughly with $0.3 \% \mathrm{NaCl} / 0.005 \mathrm{M}$ Tris (TBS). To block nonspecific binding of antisera, the sections were incubated in TBS containing $5 \%$ human serum albumin (HSA; Sigma-Aldrich RBI) and 0.005\% Tergitol type NP-10 (Sigma-Aldrich) for 30 minutes. After blocking, sections were incubated overnight in a primary antibody solution consisting of anti-CLDN4 (1:40) or anti-JAM-A (1:20) and 2\% HSA in TBS. Sections were washed thoroughly with TBS, then blocked in $2 \%$ HSA in TBS. To localize CLDN4 and JAM-A, sections were incubated for 2 hours in a solution containing a 1:40 dilution of $\mathrm{F}\left(\mathrm{ab}^{\prime}\right)_{2}$ fragment of IgG (goat anti-mouse and goat anti-rabbit, respectively), conjugated to 10-nm gold particles (Electron Microscopy Sciences), $2 \% \mathrm{HSA}$, and $5 \mathrm{mg} / \mathrm{ml}$ polyethylene glycol in TBS. After the sections were thoroughly washed and dried, they were counterstained with $1 \%$ uranyl acetate in distilled water for 45 minutes. Electron micrographs were captured at $75 \mathrm{kV}$ with a Hitachi $\mathrm{H}-7700$ electron microscope (Hitachi High Technologies America Inc.) equipped with an AMT Advantage CCD camera.

Morphometric analysis. Morphometric analyses were carried out using NIH ImageJ software. For studies in vivo, pixels positive for $\mathrm{CD} 11 \mathrm{~b}$ and claudin proteins, MBP, fibrinogen, and IgG immunoreactivity or cells positive for CD4, CD45, Olig2, or GFAP were counted by a blinded observer in projections from cortical or spinal cord sections. The same number of images was quantified from at least 5 animals per condition per genotype per time point and 5 age- and sexmatched normal controls unless otherwise indicated, and at least 4 random $\times 10$ to $\times 20$ fields in 5 sections per animal. Neuronal loss and myelin loss were quantified using previously published analyses (29); $\mathrm{NeuN}^{-}$or $\mathrm{MBP}^{-}$pixels were subtracted from the total in each field in matched projections at $\times 20$ magnification using Image $J$ software, and data were converted to absolute area.
Statistics. For multiple comparisons, 1-way or 2-way ANOVA followed by Bonferroni post-test was used. Student's $t$ test was used to compare 2 groups of matched samples. In all cases, $P$ less than 0.05 was considered significant. Data represent mean \pm SEM.

Study approval. Use of human cultures was discussed by the IRB at the Icahn School of Medicine at Mount Sinai (ISMMS), and approved as having exempt status. Studies using mice were approved by the IACUC at the ISMMS, and adhered to the American Veterinary Medical Association guidelines. The ISMMS has an Animal Welfare Assurance on file with the Office for Laboratory Animal Welfare (Assurance no. A3111-01).

\section{Author contributions}

SH, CC, ATA, and GRJ designed research studies. SH, CC, AT, KK, AG, ATA, SM, SS, YH, and JNM conducted experiments and acquired data. SH, CC, ATA, SM, YH, and GRJ analyzed data. PF, $\mathrm{ZB}, \mathrm{EDC}$, and MVS provided reagents and helped edit the manuscript. SH, CC, MVS, and GRJ wrote the manuscript.

\section{Acknowledgments}

This work was supported by National Institute of Neurological Disorders and Stroke (NINDS) research grants R01NS062703-06 and R01NS085103-01 (to GRJ), NINDS R01NS084030-03 (to MVS), National Heart, Lung, and Blood Institute (NHLBI) R01HL112638-04, R01HL126877-01A1, and P01HL119165-02 (to ZB) and U01HL108634-05 (to EDC), and NIH R24CA095823 (to the ISMMS Microscopy Facility). SH is the recipient of research residency training grant funding from NINDS R25NS079102-05, and a Leon Levy Fellowship in Neuroscience. Additional support was provided by National MS Society research grants RG5024 and RG-1501-02870 (to GRJ). This work was also supported by the ISMMS Graduate School (to JNM), and by the Beker Foundation, the Muzio Foundation, the Noto Foundation, and the Guthy-Jackson Charitable Foundation (to GRJ). EDC is Hastings Professor and Norris Chair of Medicine and receives support from the Whittier Foundation. ZB is Ralph Edgington Chair in Medicine and receives support from the Hastings Foundation.

Address correspondence to: Candice Chapouly or Gareth R. John, Annenberg 14-86, Icahn School of Medicine at Mount Sinai, 1 Gustave L. Levy Place, New York, New York 10029-6574, USA. Phone: 212.241.8142. Email: candice.chapouly@mssm.edu (C. Chapouly); gareth.john@mssm.edu (G.R. John).
1. Brück W, Bitsch A, Kolenda H, Brück Y, Stiefel $\mathrm{M}$, Lassmann H. Inflammatory central nervous system demyelination: correlation of magnetic resonance imaging findings with lesion pathology. Ann Neurol. 1997;42(5):783-793.

2. Miller DH, et al. Serial gadolinium enhanced magnetic resonance imaging in multiple sclerosis. Brain. 1988;111 ( Pt 4):927-939.

3. Miller DH, et al. High dose steroids in acute relapses of multiple sclerosis: MRI evidence for a possible mechanism of therapeutic effect. J Neurol Neurosurg Psychiatr. 1992;55(6):450-453.

4. Beck RW, et al. A randomized, controlled trial of corticosteroids in the treatment of acute optic neuritis. The Optic Neuritis Study Group. N Engl J Med.1992;326(9):581-588.

5. Mahad DH, Trapp BD, Lassmann H. Pathological mechanisms in progressive multiple sclerosis. Lancet Neurol. 2015;14(2):183-193.

6. Obermeier B, Daneman R, Ransohoff RM. Development, maintenance and disruption of the blood-brain barrier. Nat Med. 2013;19(12):1584-1596.

7. Chow BW, Gu C. The molecular constituents of the blood-brain barrier. Trends Neurosci. 2015;38(10):598-608.

8. Engelhardt B, Ransohoff RM. Capture, crawl, cross: the $\mathrm{T}$ cell code to breach the blood-brain barriers. Trends Immunol. 2012;33(12):579-589.

9. Sofroniew MV. Astrocyte barriers to neurotoxic inflammation. Nat Rev Neurosci. 2015;16(5):249-263.

10. Ciccarelli O, et al. Pathogenesis of multiple sclerosis: insights from molecular and metabolic imaging. Lancet Neurol. 2014;13(8):807-822.

11. Prakash R, Carmichael ST. Blood-brain barrier breakdown and neovascularization processes after stroke and traumatic brain injury. Curr Opin Neurol. 2015;28(6):556-564.

12. Dallasta LM, et al. Blood-brain barrier tight junction disruption in human immunodeficiency virus-1 encephalitis. Am J Pathol. 1999;155(6):1915-1927.

13. Alvarez JI, et al. The Hedgehog pathway promotes blood-brain barrier integrity and CNS immune quiescence. Science. 2011;334(6063):1727-1731.

14. Bell RD, et al. Pericytes control key neurovascular functions and neuronal phenotype in the adult brain and during brain aging. Neuron . 2010;68(3):409-427. 
15. Daneman R, Zhou L, Kebede AA, Barres BA. Pericytes are required for blood-brain barrier integrity during embryogenesis. Nature. 2010;468(7323):562-566.

16. Argaw AT, et al. Astrocyte-derived VEGF-A drives blood-brain barrier disruption in CNS inflammatory disease. JClin Invest. 2012;122(7):2454-2468.

17. Proescholdt MA, et al. Vascular endothelial growth factor (VEGF) modulates vascular permeability and inflammation in rat brain. J Neuropathol Exp Neurol. 1999;58(6):613-627.

18. Argaw AT, Gurfein BT, Zhang Y, Zameer A, John GR. VEGF-mediated disruption of endothelial CLN-5 promotes blood-brain barrier breakdown. Proc Natl Acad Sci U S A. 2009;106(6):1977-1982.

19. Chapouly C, et al. Astrocytic TYMP and VEGFA drive blood-brain barrier opening in inflammatory central nervous system lesions. Brain. 2015;138(Pt 6):1548-1567.

20. Bush TG, et al. Leukocyte infiltration, neuronal degeneration, and neurite outgrowth after ablation of scar-forming, reactive astrocytes in adult transgenic mice. Neuron. 1999;23(2):297-308.

21. Hewitt KJ, Agarwal R, Morin PJ. The claudin gene family: expression in normal and neoplastic tissues. BMC Cancer. 2006;6:186.

22. Acharya $P$, et al. Distribution of the tight junction proteins ZO-1, occludin, and claudin-4, -8 , and -12 in bladder epithelium. Am J Physiol Renal Physiol. 2004;287(2):F305-F318.

23. Furuse $\mathrm{M}$, et al. Claudin-based tight junctions are crucial for the mammalian epidermal barrier: a lesson from claudin-1-deficient mice. J Cell Biol. 2002;156(6):1099-1111.

24. Herx LM, Yong VW. Interleukin-1 beta is required for the early evolution of reactive astrogliosis following CNS lesion. J Neuropathol Exp Neurol. 2001;60(10):961-971.

25. Duffy HS, John GR, Lee SC, Brosnan CF, Spray DC. Reciprocal regulation of the junctional proteins claudin- 1 and connexin 43 by interleukin1 beta in primary human fetal astrocytes. J Neurosci. 2000;20(23):RC114.

26. Tsukita S, Furuse M. The structure and function of claudins, cell adhesion molecules at tight junctions. Ann N Y Acad Sci. 2000;915:129-135.

27. Severson EA, Parkos CA. Structural determinants of Junctional Adhesion Molecule A (JAM-A) function and mechanisms of intracellular signaling. Curr Opin Cell Biol. 2009;21(5):701-707.

28. Van Itallie CM, Anderson JM. Architecture of tight junctions and principles of molecular composition. Semin Cell Dev Biol. 2014;36:157-165.

29. Gurfein BT, et al. IL-11 regulates autoimmune demyelination. JImmunol. 2009;183(7):4229-4240.

30. Lambert D, O'Neill CA, Padfield PJ. Methylbeta-cyclodextrin increases permeability of Caco- 2 cell monolayers by displacing specific claudins from cholesterol rich domains associated with tight junctions. Cell Physiol Biochem. 2007;20(5):495-506.

31. Wanner IB, et al. Glial scar borders are formed by newly proliferated, elongated astrocytes that interact to corral inflammatory and fibrotic cells via STAT3-dependent mechanisms after spinal cord injury. J Neurosci. 2013;33(31):12870-12886

32. John GR, Chen L, Rivieccio MA, Melendez-
Vasquez CV, Hartley A, Brosnan CF. Interleukin-1beta induces a reactive astroglial phenotype via deactivation of the Rho GTPase-Rock axis. J Neurosci. 2004;24(11):2837-2845.

33. Kage $\mathrm{H}$, et al. Claudin 4 knockout mice: normal physiological phenotype with increased susceptibility to lung injury. Am J Physiol Lung Cell Mol Physiol. 2014;307(7):L524-L536.

34. Garcia AD, Doan NB, Imura T, Bush TG, Sofroniew MV. GFAP-expressing progenitors are the principal source of constitutive neurogenesis in adult mouse forebrain. Nat Neurosci. 2004;7(11):1233-1241.

35. Adams RA, et al. The fibrin-derived gamma377395 peptide inhibits microglia activation and suppresses relapsing paralysis in central nervous system autoimmune disease. J Exp Med 2007;204(3):571-582.

36. Schachtrup C, et al. Fibrinogen inhibits neurite outgrowth via beta 3 integrin-mediated phosphorylation of the EGF receptor. Proc Natl Acad Sci U S A. 2007;104(28):11814-11819.

37. Schluesener HJ, Sobel RA, Linington C, Weiner HL. A monoclonal antibody against a myelin oligodendrocyte glycoprotein induces relapses and demyelination in central nervous system autoimmune disease. JImmunol. 1987;139(12):4016-4021.

38. Lennon VA, Kryzer TJ, Pittock SJ, Verkman AS, Hinson SR. IgG marker of optic-spinal multiple sclerosis binds to the aquaporin- 4 water channel. JExp Med. 2005;202(4):473-477.

39. Song J, et al. Focal MMP-2 and MMP-9 activity at the blood-brain barrier promotes chemokineinduced leukocyte migration. Cell Rep. 2015;10(7):1040-1054.

40. Kebir H, et al. Human TH17 lymphocytes promote blood-brain barrier disruption and central nervous system inflammation. Nat Med. 2007;13(10):1173-1175.

41. Sutton C, Brereton C, Keogh B, Mills KH, Lavelle EC. A crucial role for interleukin (IL)- 1 in the induction of IL-17-producing T cells that mediate autoimmune encephalomyelitis. J Exp Med. 2006;203(7):1685-1691.

42. Badovinac V, Mostarica-Stojković M, Dinarello CA, Stosić-Grujicić S. Interleukin-1 receptor antagonist suppresses experimental autoimmune encephalomyelitis (EAE) in rats by influencing the activation and proliferation of encephalitogenic cells. J Neuroimmunol. 1998;85(1):87-95.

43. Chung Y, et al. Critical regulation of early Th17 cell differentiation by interleukin-1 signaling. Immunity. 2009;30(4):576-587.

44. Coll RC, et al. A small-molecule inhibitor of the NLRP3 inflammasome for the treatment of inflammatory diseases. Nat Med. 2015;21(3):248-255.

45. de Jong BA, et al. Production of IL-1beta and IL-1Ra as risk factors for susceptibility and progression of relapse-onset multiple sclerosis. J Neuroimmunol. 2002;126(1-2):172-179.

46. Bernatsky S, Renoux C, Suissa S. Demyelinating events in rheumatoid arthritis after drug exposures. Ann Rheum Dis. 2010;69(9):1691-1693.

47. Prins $\mathrm{M}$, et al. Interleukin-1 $\beta$ and interleukin-1 receptor antagonist appear in grey matter additionally to white matter lesions during experimental multiple sclerosis. PLoS One. 2013;8(12):e83835.
48. Cannella B, Raine CS. The adhesion molecule and cytokine profile of multiple sclerosis lesions. Ann Neurol. 1995;37(4):424-435.

49. Argaw AT, et al. IL-1beta regulates blood-brain barrier permeability via reactivation of the hypoxia-angiogenesis program. J Immunol. 2006;177(8):5574-5584.

50. Kuruvilla AP, Shah R, Hochwald GM, Liggitt HD, Palladino MA, Thorbecke GJ. Protective effect of transforming growth factor beta 1 on experimental autoimmune diseases in mice. Proc Natl Acad Sci U S A. 1991;88(7):2918-2921.

51. Luo J, et al. Glia-dependent TGF-beta signaling, acting independently of the TH17 pathway, is critical for initiation of murine autoimmune encephalomyelitis. JClin Invest. 2007;117(11):3306-3315.

52. Schachtrup C, et al. Fibrinogen triggers astrocyte scar formation by promoting the availability of active TGF-beta after vascular damage. J Neurosci. 2010;30(17):5843-5854.

53. Fujita H, Hamazaki Y, Noda Y, Oshima M, Minato N. Claudin-4 deficiency results in urothelial hyperplasia and lethal hydronephrosis. PLoS One. 2012;7(12):e52272.

54. Gong Y, et al. The Cap1-claudin-4 regulatory pathway is important for renal chloride reabsorption and blood pressure regulation. Proc Natl Acad Sci U S A. 2014;111(36):E3766-E3774.

55. Cera MR, et al. Increased DC trafficking to lymph nodes and contact hypersensitivity in junctional adhesion molecule-A-deficient mice. JClin Invest. 2004;114(5):729-738.

56. Wojcikiewicz EP, et al. LFA-1 binding destabilizes the JAM-A homophilic interaction during leukocyte transmigration. Biophys J. 2009;96(1):285-293.

57. Sladojevic $\mathrm{N}$, et al. Inhibition of junctional adhesion molecule-A/LFA interaction attenuates leukocyte trafficking and inflammation in brain ischemia/ reperfusion injury. Neurobiol Dis. 2014;67:57-70.

58. Stamatovic SM, Sladojevic N, Keep RF, Andjelkovic AV. Relocalization of junctional adhesion molecule A during inflammatory stimulation of brain endothelial cells. Mol Cell Biol. 2012;32(17):3414-3427.

59. Mayo L, Quintana FJ, Weiner HL. The innate immune system in demyelinating disease. Immunol Rev. 2012;248(1):170-187.

60. Dubois B, et al. Resistance of young gelatinase B-deficient mice to experimental autoimmune encephalomyelitis and necrotizing tail lesions. J Clin Invest. 1999;104(11):1507-1515.

61. Agrawal S, et al. Dystroglycan is selectively cleaved at the parenchymal basement membrane at sites of leukocyte extravasation in experimental autoimmune encephalomyelitis. J Exp Med. 2006;203(4):1007-1019.

62. Ma X, et al. Berberine attenuates experimental autoimmune encephalomyelitis in C57 BL/6 mice. PLoS One. 2010;5(10):e13489.

63. Gerwien $\mathrm{H}$, et al. Imaging matrix metalloproteinase activity in multiple sclerosis as a specific marker of leukocyte penetration of the blood-brain barrier. Sci Transl Med. 2016;8(364):364ra152.

64. Bruewer M, et al. Proinflammatory cytokines disrupt epithelial barrier function by apoptosisindependent mechanisms. JImmunol. 2003;171(11):6164-6172. 
65. Kinugasa T, Sakaguchi T, Gu X, Reinecker HC. Claudins regulate the intestinal barrier in response to immune mediators. Gastroenterology. 2000;118(6):1001-1011.

66. Fujita H, et al. Claudin-1 expression in airway smooth muscle exacerbates airway remodeling in asthmatic subjects. J Allergy Clin Immunol. 2011;127(6):1612-21.e8.

67. Liu J, Zhao ML, Brosnan CF, Lee SC. Expression of type II nitric oxide synthase in primary human astrocytes and microglia: role of IL-1beta and IL-1 receptor antagonist. J Immunol. 1996;157(8):3569-3576.

68. Moyon S, et al. Demyelination causes adult CNS progenitors to revert to an immature state and express immune cues that support their migration. J Neurosci. 2015;35(1):4-20.

69. Hara Y, et al. Synaptic distributions of GluA2 and PKM $\zeta$ in the monkey dentate gyrus and their relationships with aging and memory. J Neurosi. 2012;32(21):7336-7344 